\title{
Poliquetos bentónicos submareales de fondos blandos de la región de Aysén, Chile: Clado Phyllodocida (Annelida, Polychaeta)
}

\author{
Nicolás Rozbaczyloํㅜ Rodrigo A. Moreno ${ }^{1}$ \& Oscar Díaz-Díaz ${ }^{2}$ \\ ${ }^{1}$ Departamento de Ecología, Facultad de Ciencias Biológicas, \\ Pontificia Universidad Católica de Chile, Casilla 114-D, Santiago \\ ${ }^{2}$ Instituto Oceanográfico de Venezuela, Universidad de Oriente, Cumaná, Venezuela
}

\begin{abstract}
RESUMEN. Se presentan los resultados del análisis taxonómico de los poliquetos bentónicos submareales del clado Phyllodocida obtenidos durante los cruceros de investigación Cimar-7 y Cimar-8 Fiordos. Se incluye sinonimia, distribución, observaciones morfológicas e ilustraciones de cada especie. Las muestras fueron obtenidas en la región de Aysén, Pacífico suroriental frente a Chile, en fondos de limo-arcilla, mediante una rastra Agassiz entre 20 y $444 \mathrm{~m}$ de profundidad. Se recolectó un total de 119 ejemplares de poliquetos del clado Phyllodocida pertenecientes a 15 especies agrupadas en 12 géneros y seis familias. Adicionalmente, se encontró en las muestras un trozo de un ejemplar de la familia Syllidae, el que fue incluido solamente como información de registro taxonómico. Se determinó un total de siete especies de Polynoidae (46,7\% de abundancia en las muestras), cuatro especies de Nereididae (26,7\%), y una de Aphroditidae, Glyceridae, Nephtyidae y Sigalionidae (6,7\%), respectivamente. Durante el crucero Cimar-7, se recolectó un total de 67 ejemplares de poliquetos, destacándose el Sigalionidae, Leanira quatrefagesi (Kinberg) con una abundancia del 29,9\%. En el crucero Cimar-8, se recolectaron 52 poliquetos, destacándose nuevamente Leanira quatrefagesi con un 42,3\%, seguido del Polynoidae Harmothoe spinosa (Kinberg) con un 40,4\%. Estos hallazgos permiten aumentar a 66 las especies de poliquetos bentónicos submareales registrados en la región de Aysén.
\end{abstract}

Palabras clave: Polychaeta, aguas templadas frías, Provincia Magallánica, Pacífico suroriental, Chile.

\section{Benthic subtidal polychaetes on soft bottoms of the Aysén region of Chile: Clade Phyllodocida (Annelida, Polychaeta)}

\begin{abstract}
Results from a taxonomic analysis of benthic polychaetes of the clade Phyllodocida, obtained during the Cimar-7 and Cimar-8 Fjords research cruises are presented. Synonymy, distribution, morphological remarks, and illustrations for each species are included. The samples were obtained in the region of Aysén, on the southern coast of Chile, from silt-clay bottoms using an Agassiz trawl between 20 and $444 \mathrm{~m}$ depth. A total of 119 specimens of polychaetes from the clade Phyllodocida were collected. These specimens consisted of 15 species, grouped into 12 genera and 6 families. One incomplete specimen of Syllidae recovered was included only for the taxonomic record. The samples analyzed included a total of seven species of Polynoidae (46.7\% of total abundance of all samples), four species of Nereididae (26.7\% abundance) and one each of the Aphroditidae, Sigalionidae, Glyceridae and Nephtyidae (6.7\% abundance). During the Cimar-7 cruise a total of 67 specimens of polychaetes were collected, including the sigalionid Leanira quatrefagesi (Kinberg) which accounted for $29.9 \%$ of the total abundance. During the Cimar- 8 cruise, 52 specimens of polychaetes were collected, in particular Leanira quatrefagesi accounting for $42.3 \%$ of total abundance, followed by the polynoid Harmothoe spinosa (Kinberg) with $40.4 \%$ abundance. Our findings increased the number of benthic polychaetes recorded for the Aysén region to 66 species.
\end{abstract}

Key words: Polychaeta, cold temperate waters, Magellanic Province, SE Pacific, Chile. 


\section{INTRODUCCIÓN}

A nivel mundial, el clado Phyllodocida está integrado por 28 familias y más de 2000 especies (Rouse $\&$ Pleijel, 2001). La evidencia de monofilia se basa en la presencia de palpos sensoriales en posición ventral, cirros tentaculares, probóscide muscular axial, quetas compuestas por ligamentos simples y la pérdida de pliegues dorso-laterales (Fauchald \& Rouse, 1997; Rouse, 2000).

Los miembros del clado, se caracterizan por poseer palpos como órganos sensitivos, un par de antenas, faringe hipertrofiada cilíndrica, eversible, y uno o dos pares de mandíbulas. En este grupo se presenta una gran variedad de estrategias tróficas, como especies carnívoras, herbívoras, detritívoras, omnívoras y endoparásitas (Myzostomidae) (Glasby et al., 2000; Rouse \& Pleijel, 2001). Varias familias presentan niveles de cefalización variable. En Chrysopetalidae, la función locomotora de los notópodos ha cambiado a defensiva y en Tomopteridae, Lopadorhynchidae, y algunas especies de la familia Phyllodocidae, los parápodos se han modificado para realizar función natatoria (Rouse \& Pleijel, 2001).

En la costa del Pacífico suroriental frente a Chile continental, se han registrado hasta ahora 165 especies bentónicas del clado Phyllodocida distribuidas en 14 familias, asociadas principalmente a ambientes de fondos blandos (Rozbaczylo, 1985). En la zona austral de Chile (i.e. fiordos magallánicos), frente a la región de Aysén, toda la información existente para este clado proviene principalmente de expediciones extranjeras que visitaron la costa hace más de 40 años (Wesenberg-Lund, 1962; Hartmann-Schröder, 1962b, 1965) y de un estudio de Cañete et al. (1999) en el fiordo Aysén.

Con el desarrollo de los programas de investigación Cimar-7 y Cimar-8 Fiordos entre los años 2001 y 2002 en la región de Aysén, se han podido incrementar las investigaciones en estas áreas remotas y de difícil acceso de la costa de Chile, contribuyendo a generar nuevos conocimientos científicos del ambiente marino y sus recursos. De esta forma, para la fauna de poliquetos bentónicos de la región de Aysén se han registrado hasta el momento seis clados (Phyllodocida, Terebellida, Eunicida, Scolecida, Sabellida y Spionida), en fondos blandos submareales del mar interno de esta región. El objetivo del presente trabajo es dar a conocer la fauna de poliquetos bentónicos submareales del clado Phyllodocida recolectados en muestras bentónicas obtenidas en la región de Aysén y entregar antecedentes taxonómicos de cada especie.

\section{MATERIALES Y MÉTODOS}

Los poliquetos se obtuvieron como parte de muestras bentónicas recolectadas en los cruceros Cimar7 y Cimar-8 Fiordos, desde la boca del Guafo $\left(43^{\circ} 45^{\prime} \mathrm{S}\right)$ al estero Elefante $\left(46^{\circ} 28^{\prime} \mathrm{S}\right)$, en julio de 2001, a profundidades entre 20 y 444 m (Tabla 1) y desde boca del Guafo a bahía Anna Pink (4549'S), en julio de 2002, a profundidades entre 62 y 345 m, respectivamente (Tabla 2), con el buque oceanográfico AGOR "Vidal Gormaz".

Las muestras fueron obtenidas con una rastra Agassiz de 1,4 $\mathrm{m}$ de apertura de boca y $0,5 \mathrm{~m}$ de alto, en fondos de limo-arcilla y posteriormente preservadas en una solución de formalina en agua de mar al 10\% neutralizada con bórax. Los poliquetos contenidos en cada muestra de sedimento fueron extraídos bajo lupa binocular y transferidos a una solución de etanol al 70\% para su preservación. Para la determinación taxonómica se utilizó principalmente los trabajos de Hartmann-Schröder (1962b, 1965), Fauchald (1977a) y Rozbaczylo (1980). Todos los taxa fueron agrupados en el clado Phyllodocida, según Rouse \& Fauchald (1997).

Los ejemplares de cada especie se midieron con un ocular graduado, considerando la longitud total desde el extremo anterior del prostomio hasta el final del cuerpo y el ancho máximo desde el extremo distal de las setas opuestas de cada parápodo. En la sección de material examinado, se detalla para cada especie, el crucero Cimar de recolección, el número de la estación, número de ejemplares y código de depósito.

Ejemplares de cada especie fueron depositados en la sala "Colección de Flora y Fauna Profesor Patricio Sánchez Reyes" (SSUC), de la Facultad de Ciencias Biológicas de la Pontificia Universidad Católica de Chile, Santiago y en el Museo Nacional de Historia Natural, Santiago (MNHNCL-An).

\section{RESULTADOS}

En ambos cruceros, se recolectaron 119 ejemplares de poliquetos bentónicos submareales del clado Phyllodocida correspondientes a 15 especies, agrupados en 12 géneros y 6 familias. Adicionalmente, se encontró en las muestras un trozo de un ejemplar 
Tabla 1. Datos de las estaciones con ocurrencia de poliquetos bentónicos obtenidos en la región de Aysén, desde Boca del Guafo a estero Elefante, durante el crucero Cimar-7 Fiordos, en julio de 2001.

Table 1. Data from each of the sampling stations where benthic polychaetes were recorded at the region of Aysén, from Boca del Guafo to Estero Elefante, during the Cimar-7 Fjords cruise, July 2001.

\begin{tabular}{|c|c|c|c|c|}
\hline Estación & Localidad & Latitud (S) & Longitud (W) & Profundidad (m) \\
\hline 4 & Boca del Guafo & $43^{\circ} 39^{\prime}$ & $73^{\circ} 50^{\prime}$ & 166 \\
\hline 6 & Canal Moraleda & $43^{\circ} 59^{\prime}$ & $73^{\circ} 21^{\prime}$ & 198 \\
\hline 13 & Canal Moraleda & $45^{\circ} 16^{\prime}$ & $73^{\circ} 39^{\prime}$ & 58 \\
\hline 14 & Canal Moraleda & $45^{\circ} 21^{\prime}$ & $73^{\circ} 38^{\prime}$ & 160 \\
\hline $17 \mathrm{~A}$ & Fiordo Aysén & $45^{\circ} 17^{\prime}$ & $73^{\circ} 10^{\prime}$ & 335 \\
\hline 21 & Fiordo Aysén & $45^{\circ} 24^{\prime}$ & $72^{\circ} 51^{\prime}$ & 166 \\
\hline $21 \mathrm{~A}$ & Bahía Chacabuco & $45^{\circ} 28^{\prime}$ & $72^{\circ} 49^{\prime}$ & 100 \\
\hline 22 & Canal Costa & $45^{\circ} 29^{\prime}$ & $73^{\circ} 31^{\prime}$ & 304 \\
\hline 24 & Estero Elefante & $45^{\circ} 51^{\prime}$ & $73^{\circ} 35^{\prime}$ & 120 \\
\hline 25 & Estero Elefante & $46^{\circ} 05^{\prime}$ & $73^{\circ} 37^{\prime}$ & 50 \\
\hline 26 & Estero Elefante & $46^{\circ} 19^{\prime}$ & $73^{\circ} 42^{\prime}$ & 20 \\
\hline 27 & Estero Elefante & $46^{\circ} 28^{\prime}$ & $73^{\circ} 48^{\prime}$ & 120 \\
\hline 29 & Estero Quitralco & $45^{\circ} 46^{\prime}$ & $73^{\circ} 30^{\prime}$ & 120 \\
\hline 32 & Canal Jacaf & $44^{\circ} 17^{\prime}$ & $73^{\circ} 10^{\prime}$ & 444 \\
\hline 37 & Ventisquero & $44^{\circ} 31^{\prime}$ & $72^{\circ} 40^{\prime}$ & 250 \\
\hline 41 & Canal Puyuhuapi & $44^{\circ} 53^{\prime}$ & $73^{\circ} 02^{\prime}$ & 237 \\
\hline
\end{tabular}

Tabla 2. Datos de las estaciones con ocurrencia de poliquetos bentónicos obtenidos en la región de Aysén, desde Boca del Guafo a bahía Anna Pink, durante el crucero Cimar-8 Fiordos, en julio de 2002.

Table 2. Data from each of the sampling stations where benthic polychaetes were recorded at the region of Aysén, from Boca del Guafo to Anna Pink Bay, during the Cimar-8 Fjords cruise, July 2002.

\begin{tabular}{|ccccc|}
\hline Estación & Localidad & Latitud $(\mathbf{S})$ & Longitud $(\mathbf{W})$ & Profundidad (m) \\
\hline 1 & Boca del Guafo & $43^{\circ} 45^{\prime}$ & $74^{\circ} 36^{\prime}$ & 230 \\
4 & Boca del Guafo & $43^{\circ} 39^{\prime}$ & $73^{\circ} 51^{\prime}$ & 200 \\
6 & frente a isla Refugio & $43^{\circ} 59^{\prime}$ & $73^{\circ} 21^{\prime}$ & 230 \\
8 & Canal Moraleda & $44^{\circ} 26^{\prime}$ & $73^{\circ} 27^{\prime}$, & 200 \\
9 & Canal Moraleda & $44^{\circ} 41^{\prime}$ & $73^{\circ} 30^{\prime}$ & 345 \\
14 & Canal Moraleda & $45^{\circ} 22^{\prime}$ & $73^{\circ} 40^{\prime}$ & 75 \\
43 & isla Melinka & $43^{\circ} 54^{\prime}$ & $73^{\circ} 42^{\prime}$ & 64 \\
46 & Boca del Canal Tuamapu & $43^{\circ} 58^{\prime}$ & $74^{\circ} 19^{\prime}$ & 160 \\
49 & Canal Tuamapu & $44^{\circ} 28^{\prime}$ & $73^{\circ} 51^{\prime}$ & 220 \\
51 & Canal King & $44^{\circ} 34^{\prime}$, & $74^{\circ} 08^{\prime}$, & 180 \\
53 & Canal King & $44^{\circ} 35^{\prime}$ & $74^{\circ} 27^{\prime}$ & 180 \\
54 & Canal Memory & $44^{\circ} 44^{\prime}$ & $74^{\circ} 22^{\prime}$ & 246 \\
59 & Canal Ninualac & $44^{\circ} 58^{\prime}$ & $73^{\circ} 51^{\prime}$ & 180 \\
61 & Canal Ninualac & $45^{\circ} 01^{\prime}$ & $74^{\circ} 07^{\prime}$ & 275 \\
63 & Canal Ninualac & $45^{\circ} 02^{\prime}$ & $74^{\circ} 21^{\prime}$ & 160 \\
65 & Canal Darwin & $45^{\circ} 26^{\prime}$ & $74^{\circ} 21^{\prime}$ & 180 \\
67 & Canal Darwin & $45^{\circ} 23^{\prime}$ & $74^{\circ} 06^{\prime}$ & 167 \\
71 & Canal Chacabuco & $45^{\circ} 43^{\prime}$ & $74^{\circ} 03^{\prime}$ & 70 \\
72 & Canal Chacabuco & $45^{\circ} 42^{\prime}$ & $74^{\circ} 10^{\prime}$ & 62 \\
76 & Canal Pulluche & $45^{\circ} 49^{\prime}$ & $74^{\circ} 32^{\prime}$ & 192 \\
78 & Bahía Anna Pink & $45^{\circ} 49^{\prime}$ & $74^{\circ} 53^{\prime}$ & 80 \\
\hline
\end{tabular}


de la familia Syllidae, el que fue incluido solamente como información taxonómica. En el conjunto de muestras analizadas se determinó un total de siete especies de Polynoidae (46,7\% de abundancia en las muestras), cuatro especies de Nereididae (26,7\%), y una de Aphroditidae, Sigalionidae, Glyceridae y Nephtyidae (6,7\%), respectivamente. Durante el crucero Cimar-7, se recolectó un total de 67 poliquetos del clado Phyllodocida, destacándose un representante de la familia Sigalionidae, Leanira quatrefagesi (Kinberg) con una abundancia del 29,9\% (Tabla 3). En el crucero Cimar-8, se recolectaron 52 poliquetos, destacándose nuevamente Leanira quatrefagesi con un $42,3 \%$, seguido del Polynoidae Harmothoe spinosa (Kinberg) con un $40,4 \%$ (Tabla 3). Con estos resultados aumenta a 66 el número de especies de poliquetos bentónicos registradas en la región de Aysén.

\section{APHRODITIDAE Malmgren}

Aphrodita bisetosa Rozbaczylo \& Canahuire, 2000 (Figs. 1a-1g)

Aphrodita bisetosa Rozbaczylo \& Canahuire, 2000: 493-499, figs. 2-3.

Material examinado: Cimar-7: E-21 (1 ejemplar, SSUC 7108).

Caracterización: cuerpo ovalado y arqueado dorsalmente, de $61 \mathrm{~mm}$ de longitud y $29 \mathrm{~mm}$ de ancho, con 38 setígeros. Dorso cubierto por una felpa. Prostomio pequeño, redondeado, con un par de áreas oculares; antena media con ceratóforo basal y ceratostilo alargado, ligeramente más corto que el prostomio. Palpos bi-articulados, finamente papilados. 15 pares de élitros semitransparentes con escasas micropapilas digitiformes en su superficie; los élitros se disponen, imbricados, sobre los setígeros $2,5,7,9 \ldots .25,28,31$, cubiertos completamente por el afelpado dorsal. Papila branquial fimbriada a partir del sexto setígero sobre el margen posterolateral del tubérculo dorsal. Parápodos

Tabla 3. Especies de poliquetos bentónicos del clado Phyllodocida recolectadas en la región de Aysén, durante los cruceros Cimar-7 y Cimar-8 Fiordos, en julio de 2001 y julio de 2002. Para cada especie se presenta el número de ejemplares recolectados en cada crucero y la abundancia relativa (\%). Las abreviaciones indican: A= Aphroditidae; $\mathbf{P}=$ Polynoidae; $\mathbf{S}=$ Sigalionidae; $\mathbf{N}=$ Nereididae; $\mathbf{G}=$ Glyceridae $\mathbf{y} \mathbf{N e}=\mathbf{N e p h t y i d a e}$.

Table 3. Benthic polychaete species of the clade Phyllodocida, collected during the Cimar-7 and Cimar-8 Fjords cruises at the region of Aysén, July 2001 and July 2002. For each species we provide the number of specimens collected during each cruise and the relative abundance (\% of total). The abbreviations are: $A=A p h r o d i t i d a e ;$ $\mathbf{P}=$ Polynoidae; $\mathbf{S}=$ Sigalionidae $\mathbf{N}=$ Nereididae $\mathbf{G}=$ Glyceridae and $\mathbf{N e}=$ Nephtyidae.

\begin{tabular}{|lcccc|}
\hline Especie & Cimar-7 & Abundancia (\%) & Cimar-8 & Abundancia (\%) \\
\hline Aphrodita bisetosa $(\mathrm{A})$ & 1 & 1,5 & 0 & 0 \\
Austrolaenilla antarctica $(\mathrm{P})$ & 2 & 3,0 & 0 & 0 \\
Euphionella lobulata $(\mathrm{P})$ & 1 & 1,5 & 0 & 0 \\
Euphionella patagonica $(\mathrm{P})$ & 0 & 0 & 2 & 0 \\
Halosydna patagonica $(\mathrm{P})$ & 1 & 1,5 & 0 & 0 \\
Harmothoe cf. exanthema $(\mathrm{P})$ & 6 & 9,0 & 0 & 40,4 \\
Harmothoe spinosa $(\mathrm{P})$ & 4 & 6,0 & 21 & 0 \\
Polyeunoa dubia $(\mathrm{P})$ & 18 & 26,9 & 0 & 42,3 \\
Leanira quatrefagesi $(\mathrm{S})$ & 20 & 29,9 & 22 & 0 \\
Ceratocephale fauveli $(\mathrm{N})$ & 1 & 1,5 & 0 & 0 \\
Nereis callaona $(\mathrm{N})$ & 3 & 4,5 & 0 & 5,8 \\
Nereis eugeniae $(\mathrm{N})$ & 2 & 3,0 & 0 & 0 \\
Platynereis australis $(\mathrm{N})$ & 6 & 9,0 & 3 & 7,7 \\
Glycera papillosa $(\mathrm{G})$ & 1 & 1,5 & 0 & 100 \\
Aglaophamus peruana $(\mathrm{Ne})$ & 1 & 1,5 & 4 & 52 \\
\hline Total & 67 & 100 & & 0 \\
\hline
\end{tabular}



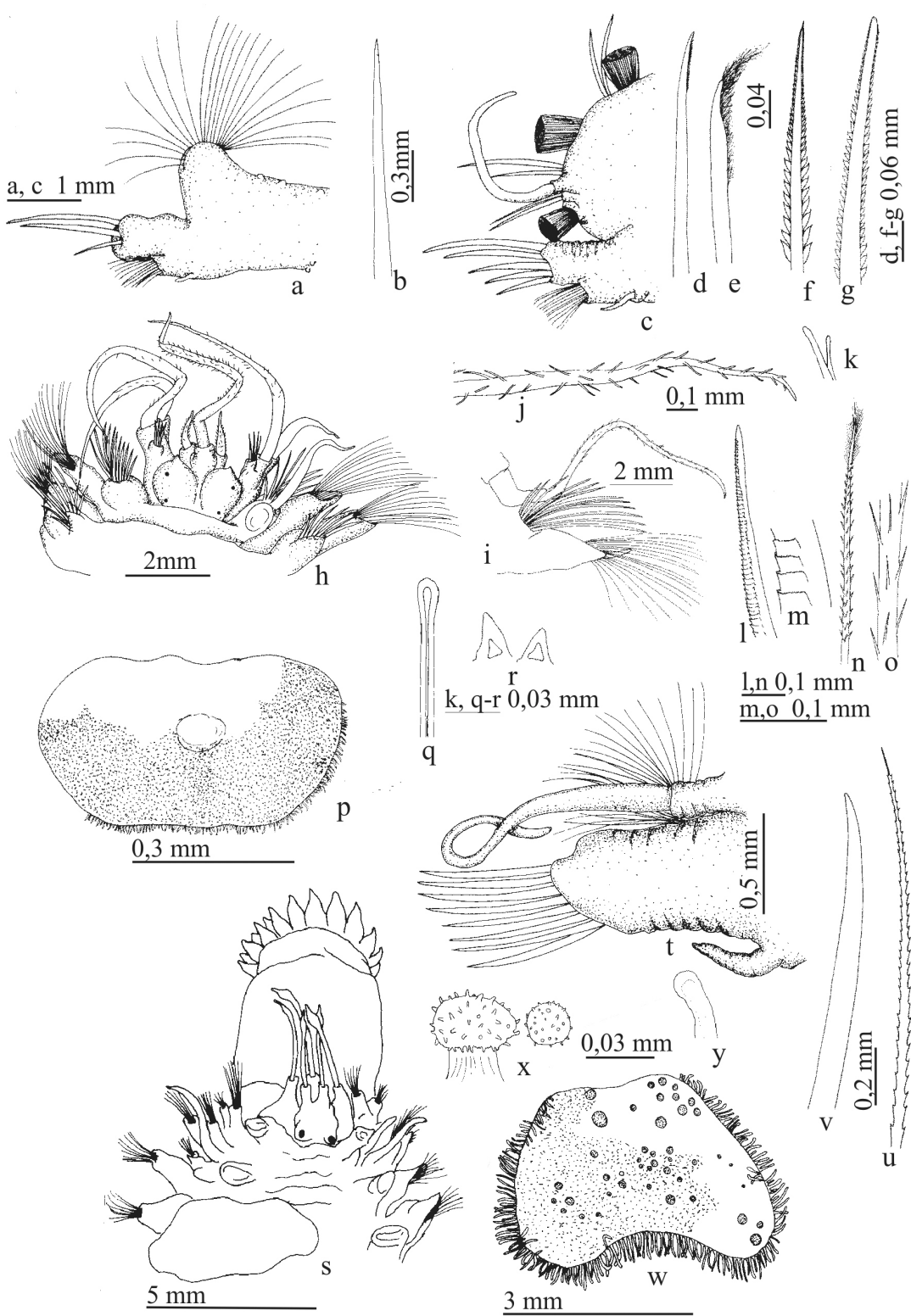

q
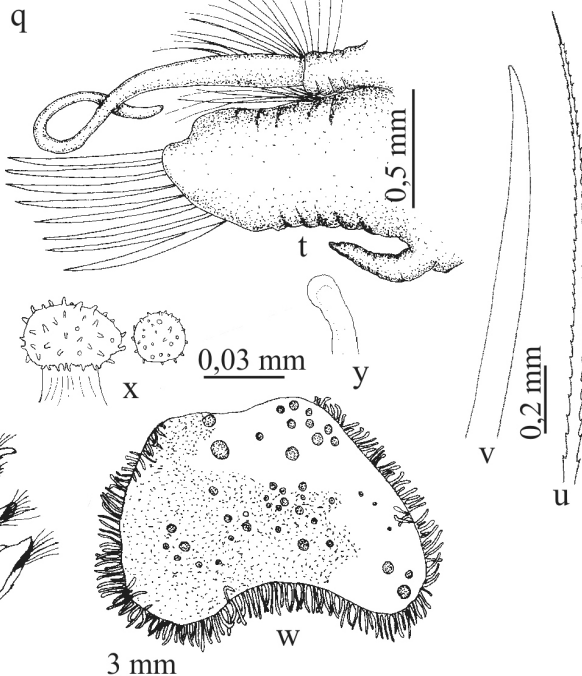

Figura 1. Aphrodita bisetosa: a) primer parápodo, b) seta acicular distalmente curvada, c) neurópodo medio, de) neurosetas aciculares, f-g) setas bipinnadas. Austrolaenilla antarctica: $\mathbf{h}$ ) extremo anterior en vista dorsal, i) parápodo medio, j) detalle del cirro dorsal, $k$ ) papilas del cirro dorsal, l-m) notosetas denticuladas, $\mathbf{n}-\mathbf{o}$ ) neurosetas espinulosas con extremo distal piloso, p) élitro, q) papila filiforme marginal, r) papilas cónicas. Euphionella patagonica: s) extremo anterior en vista dorsal mostrando la faringe evertida, t) parápodo birremo, u) notoseta capilar con procesos espinosos, $v$ ) neuroseta acicular, w) élitro, $x$ ) papilas distalmente globulares y espinosas, $y$ ) papila digitiforme distalmente ensanchada.

Figure 1. Aphrodita bisetosa: a) first parapodium, b) distally curved acicular seta, c) middle neuropodium, d-e) acicular neurosetae, f-g) bipinnate setae. Austrolaenilla antarctica: h) anterior end in dorsal view, i) middle parapodium, j) detail of dorsal cirrus, $k$ ) papillae of dorsal cirrus, l-m) denticulate notosetae, n-o) spinulose neurosetae with hairy distal end, p) elytron, q) marginal filiform papilla, r) conical papillae. Euphionella patagonica: s) anterior end in dorsal view showing everted pharynx, t) biramous parapodium, u) capillary notosetae with spinulose processes, $v$ ) acicular neurosetae, $w$ ) elytron, $x$ ) distally globular and spinulose papillae, y) distally expanded digitiform papilla. 

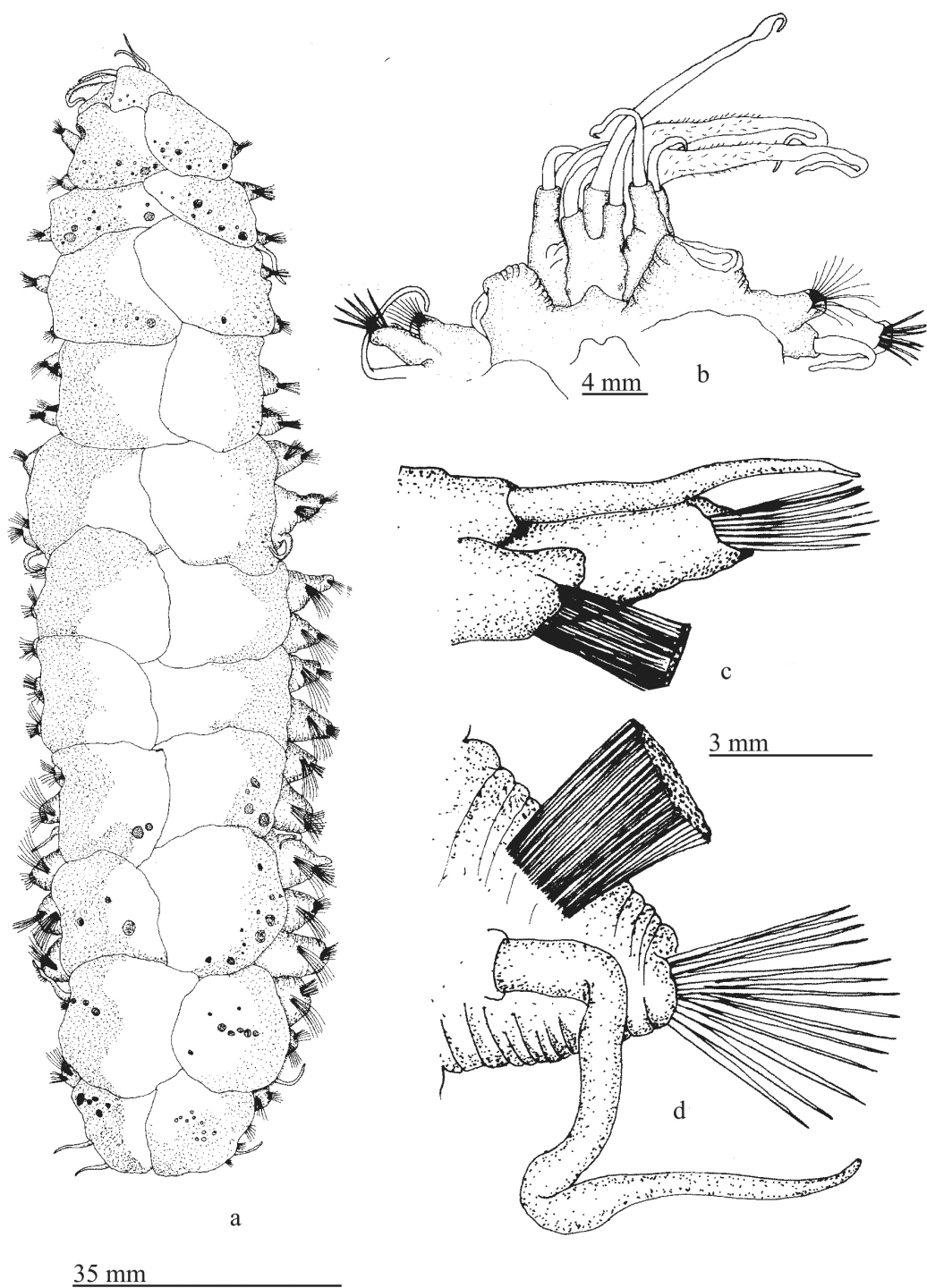

$35 \mathrm{~mm}$

Figura 2. Euphionella lobulata. a) ejemplar en vista dorsal, b) región anterior en vista dorsal, c) parápodo anterior, d) parápodo medio, en vista dorsal.

Figure 2. Euphionella lobulata. a) dorsal view of the specimen, b) anterior region in dorsal view, c) anterior parapodium, d) middle parapodium in dorsal view.

birramosos. Primer setígero (Fig. 1a) alargado y proyectado anteriormente. Notópodos rectangulares con un haz de setas muy finas, sedosas y flexibles, que conforman el afelpado dorsal y setas aciculares distalmente curvadas y de extremo romo (Fig. 1b). Neurópodos cilíndricos (Fig. 1c), con pequeñas papilas. Neurosetas aciculares (Figs. 1d yle) y un haz inferior de setas bipinnadas (Figs. 1f y $1 \mathrm{~g}$ ). Ci- rro ventral subcónico, corto, localizado cerca de la base del neurópodo. Cirro dorsal largo y liso, dirigido dorsalmente, presente sólo en los setígeros sin élitros.

Distribución geográfica: Chile, frente a Los Vilos y Papudo (Rozbaczylo \& Canahuire, 2000), fiordo Aysén (este estudio). 


\section{POLYNOIDAE Malmgren}

\section{Austrolaenilla antarctica Bergström, 1916}

(Figs. 1h-1r)

Austrolaenilla antarctica Bergström, 1916: 291-294, pl. 3, fig. 8, pl. 5, fig. 1-2; Hartman, 1953: 14; Rozbaczylo, 1985: 24.

Antinoe antarctica: Fauvel, 1936: 9-10; Monro, 1936: 96; Wesenberg-Lund, 1962: 26.

Laenilla obnupta: Fauvel, 1936: 9.

Antionella antarctica: Hartman, 1964: 15, pl. 2, fig. 5, pl. 3, figs. 6-8.

Material examinado: Cimar-7: E-27 (2 ejemplares, SSUC 7109).

Caracterización: el ejemplar de mayor tamaño mide $38 \mathrm{~mm}$ de longitud y $9 \mathrm{~mm}$ de ancho. Prostomio ovalado, con dos pares de ojos en arreglo cuadrangular (Fig. 1h). Antena media larga, con la superficie papilada, cirróforo corto; antenas laterales $1 / 5$ de la longitud de la media, insertas ventralmente, con papilas filiformes en su superficie. Parápodos birramosos (Fig. 1i), con un largo cirro dorsal papilado (Figs. 1j y 1k). Notosetas denticuladas (Figs. 11 y 1m). Neurosetas espinulosas con extremo distal piloso (Figs. 1n y 1o). Élitros transparentes con $2 / 3$ de su superficie pigmentada (Fig. 1p), con papilas filiformes marginales (Fig. 1q) y cónicas (Fig. 1r).

Distribución geográfica: isla Georgia del Sur (Bergström, 1916), Tierra de Graham (Fauvel, 1950), estrecho de Magallanes (Wesenberg-Lund, 1962), estero Elefante (este estudio), península Antártica (Fauvel, 1936) y Nueva Zelanda (Knox, 1960).

\section{Euphionella lobulata (Seidler, 1922)}

(Figs. 2a-2d, 3e-3o)

Physalidonotus lobatus Seidler, 1922: 89-91. Euphionella lobulata: Wesenberg-Lund, 1962: 16; Rozbaczylo, 1985: 27.

Material examinado: Cimar-7: E-27 (1 ejemplar, SSUC 7110).

Caracterización: cuerpo robusto, ligeramente arqueado, de $92 \mathrm{~mm}$ de longitud y $35 \mathrm{~mm}$ de ancho, con 25 setígeros (Fig. 2a). Prostomio (Fig. 2b) más largo que ancho con un par de ojos subdermales. Antena media larga y ensanchada subdistalmente; antenas laterales la mitad de longitud que la media. Palpos papilados. Parápodos birramosos; en los cua- tro primeros setígeros el lóbulo notopodial está bien desarrollado (Fig. 2c), pero a partir del quinto setígero se hace inconspicuo y el haz de setas se proyecta desde la pared dorsal al lóbulo setígero (Fig. 2d). Cirro parapodial dorsal más largo que el lóbulo setígero, el ventral es corto y cónico (Fig. 3e). Doce pares de élitros, con margen fimbriado (Fig. 3f). Elitros con papilas digitiformes (Fig. 3g), filiformes distalmente globulares (Fig. 3h), filiformes distalmente globulares espinosas (Fig. 3i), globulares (Fig. 3j) y globulares pedunculadas gruesas (Fig. $3 \mathrm{k}$ ); la mayoría de estas papilas sólo están presentes en los cuatro primeros y en los últimos pares de élitros. Pseudoélitros (Fig. 31), presentes en los segmentos cirríferos. Branquias presentes a partir del tercer setígero como una pequeña proyección digitiforme en el margen posterior del notópodo; entre el cuarto y sexto setígeros las branquias se presentan como pequeños lóbulos con la base ancha; del séptimo setígero en adelante comienzan a aparecer las proyecciones digitiformes (Fig. 3m); a partir del cuarto setígero comienzan a aparecer pequeñas papilas como pequeños lóbulos inconspicuos, que a partir del quinto se hacen evidentes. Notosetas capilares lisas (Fig. 3n). Neurosetas aciculares lisas (Fig. 3o).

Observaciones: Wesenberg-Lund (1962) señala que el único registro que se tiene de esta especie corresponde a la descripción original de Seidler (1922), basada en la porción anterior ( 9 setígeros) de un ejemplar recolectado en Calbuco. Las características del ejemplar examinado por nosotros coinciden con lo señalado por Seidler (1922). En relación con las otras dos especies de Euphionella registradas en Chile, ya se han señalado las diferencias con $E$. patagonica; con respecto a $E$. robusta, los cirros dorsales son más largos y las papilas son diferentes, el lóbulo notopodial está poco desarrollado y las notosetas son capilares muy largas y flexibles dispuestas en un haz muy numeroso.

Distribución geográfica: en Chile, Calbuco (Seidler, 1922) y Aysén (este estudio).

\section{Euphionella patagonica Monro, 1936}

(Figs. 1s-1y)

Euphionella patagonica Monro, 1936: 97-100, fig. 10; Fauvel, 1941: 276; Wesenberg-Lund, 1962: 1617; Hartman, 1964: 23, pl. 6, figs. 1-3; HartmannSchröder, 1965: 72-75, figs. 18-22; Rozbaczylo, 1985: 27. 

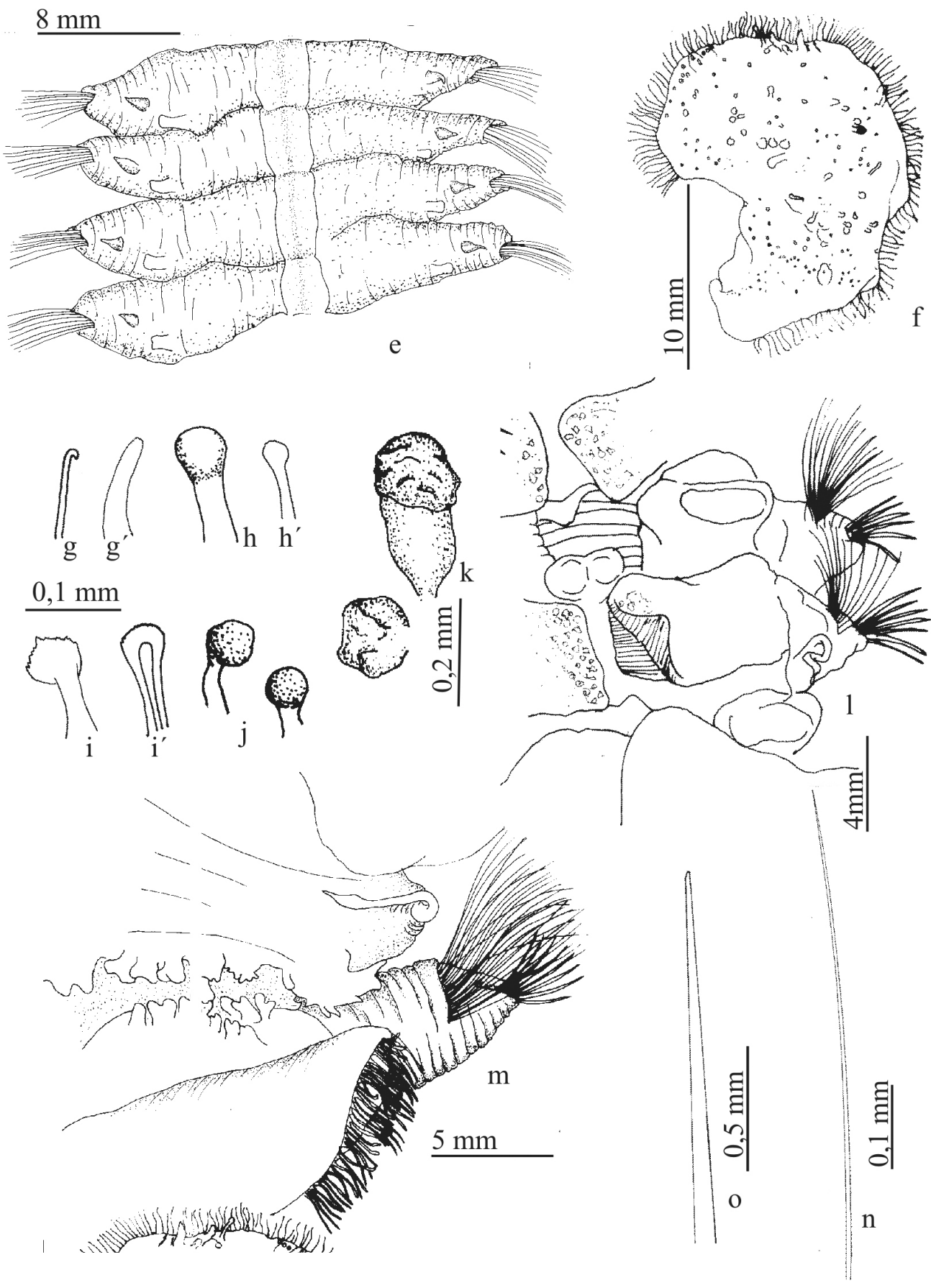

Figura 3. Euphionella lobulata. e) parápodos medios, en vista ventral, f) élitro, g) papila digitiforme, h) papila filiforme distalmente globular, i-i') papila filiforme distalmente globular espinosa, j) papilas globulares, $\mathbf{k}$ ) papilas globulares pedunculadas gruesas, I) detalle de pseudoélitros, m) branquias y papilas elitrígeras del setígero 16, n) notoseta, o) neuroseta.

Figure 3. Euphionella lobulata. e) middle parapodia in ventral view, f) elytron, g) digitiform papilla, h) distally globular smooth filiform papilla, $\mathbf{i}-\mathbf{i}$ ') distally globular spinulose filiform papilla, $\mathbf{j}$ ) globular papillae, $k$ ) wide peduncled globular papillae, I) detail of pseudoelytra, $m$ ) branchiae and papillae from setiger 16, n) notoseta, $o$ ) neuroseta. 
Material examinado: Cimar-8: E-1 (1 ejemplar, SSUC 7111), E-65 (1 ejemplar, SSUC 7112).

Caracterización: el ejemplar de mayor tamaño mide $41 \mathrm{~mm}$ de longitud y $10 \mathrm{~mm}$ de ancho, con 23 setígeros y 12 pares de élitros. Prostomio rectangular, más largo que ancho, con un surco medio inconspicuo; dos pares de ojos; antena media tres veces la longitud del prostomio y antenas laterales la mitad de longitud que la media. Faringe cilíndrica con 20 papilas distales triangulares (Fig. 1s). Palpos papilados. Segmento tentacular con pocas setas. Parápodos birramosos (Fig. 1t). El cirro parapodial dorsal es largo, el ventral es corto y no sobrepasa al lóbulo setígero. Branquias presentes desde el tercer setígero, unidas a los elitróforos. Las notosetas son capilares con procesos espinosos (Fig. 1u). Las neurosetas son aciculares (Fig. 1v). Los élitros (Fig. 1w) cubren toda la superficie dorsal del animal y están provistos con papilas distalmente globulares y espinosas (Fig. 1x), digitiformes distalmente ensanchadas (Fig. 1y) y filiformes; margen de los élitros fimbriado.

Observaciones: en comparación con las otras dos especies del género registradas en Chile, se diferencia de E. robusta Wesenberg-Lund por la forma y tamaño de las papilas y, además, en esta última especie el cirro dorsal es corto, no sobrepasado el lóbulo setígero. Respecto a E. lobulata (Seidler), además de las formas de las papilas, en ésta las notosetas son capilares lisos.

Distribución geográfica: norte de las islas Falkland (Monro, 1936). En Chile, en el golfo de Ancud (Wesenberg-Lund, 1962), Aysén (este estudio) e isla Hoste (Fauvel, 1941).

\section{Halosydna patagonica Kinberg, 1855}

(Figs. 4s-4w)

Halosydna patagonica Kinberg, 1855: 385; 18581910: 17, pl. 5, fig. 23; Ehlers, 1901a: 256; 1901b: 45-47; Seidler, 1924: 116-118; Fauvel, 1941: 276277; Hartman, 1948: 16-17, pl. 2, figs. 15-18; 1964 : 25, pl. 6, figs. 6-7; Wesenberg-Lund, 1962: 14-15; Hartmann-Schröder, 1965: 76, 78, 282; Rozbaczylo, 1985: 28-29.

Material examinado: Cimar-7: E-24 (1 ejemplar, SSUC 7113).

Caracterización: los dos ejemplares están incompletos. Parápodos birramosos (Fig. 4s); el cirro dor- sal es largo, alcanzando el extremo distal de las neurosetas. Notosetas con margen aserrado de dos tipos: largas distalmente aguzadas (Fig. 4t) y cortas distalmente redondeadas (Fig. $4 \mathrm{u}$ ). Neurosetas distalmente bidentadas (Fig. 4v), con una serie de delgados dientes en la parte subdistal. Élitros con papilas cónicas (Fig. 4w), localizadas sobre la mitad posterior del mismo.

Observaciones: aun cuando sólo se contaba con la región posterior de los ejemplares examinados, las características de las setas y las papilas permitieron reconocer la especie. $H$. patagonica es muy parecida a $H$. parva Kinberg, de la cual se diferencia por los dientes secundarios de la seta bidentada que no son tan gruesos o fuertes como en esta última especie.

Distribución geográfica: en Chile, desde Iquique hasta la boca oriental del estrecho de Magallanes (Ehlers, 1901a), estrecho de Magallanes (Kinberg, 1855, Wesenberg-Lund, 1962).

\section{Harmothoe cf. exanthema (Grube, 1856)}

(Figs. 4k-4r)

Polynoe exanthema Grube: 1856: 46.

Harmothoe exanthema: Wesenberg-Lund, 1962: 23-

24; Hartman, 1964: 28-29, pl. 7, fig. 7; Rozbaczylo, 1985: 29-30.

Lagisca vesiculosa: Ehlers, 1900: 209; 1901a: 256; 1901b: 42.

Lagisca globulosa Hartmann-Schröder, 1962b: 6265, figs. 9-15.

Lagisca exanthema: Hartmann-Schröder, 1965: 67-69.

Material examinado: Cimar-7: E-25 (2 ejemplares, SSUC 7114), E-27 (4 ejemplares, SSUC 7115).

Caracterización: los dos ejemplares están incompletos. Prostomio más ancho que largo, con 2 pequeñas proyecciones frontales y dos pares de ojos (Fig. 4k). Antena media papilada con cirróforo grueso, antenas laterales papiladas, cortas. Parápodos birramosos (Fig. 4l). Notosetas pectinadas (Fig. 4m). Neurosetas pectinadas con el extremo distal entero (Fig. 4n). Dorso cubierto por 15 pares de élitros (Fig. 4o), con micropapilas esféricas, papilas cónicas (Fig. 4p), digitiformes (Fig. 4q) y macropapilas globulares (Fig. 4r), con un pequeño pedúnculo y en el ápice un filamento filiforme largo y uno corto, a su vez la superficie globular cubierta por numerosas micropapilas. Superficie dorsal con bandas transversales oscuras. 


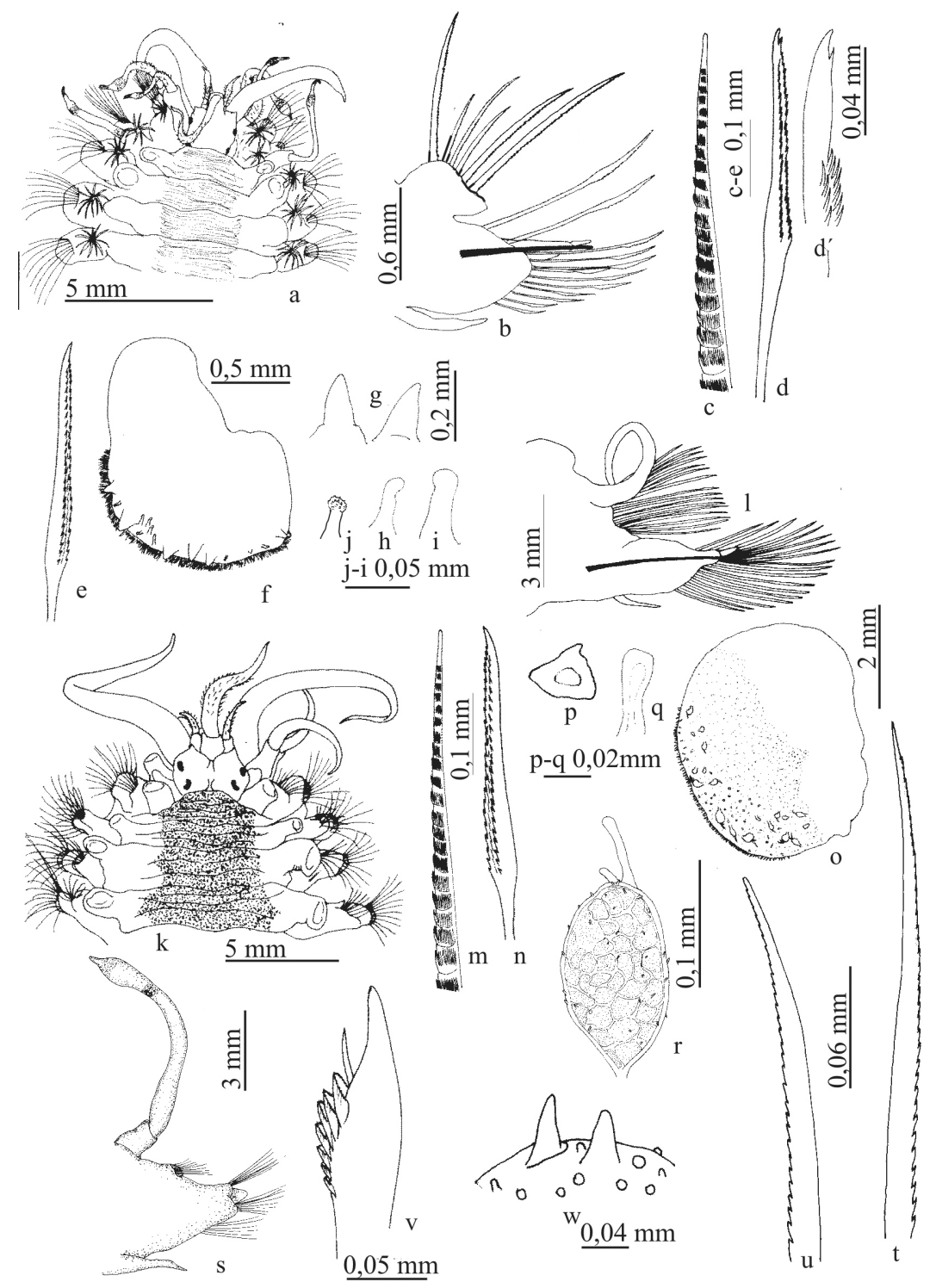

Figura 4. Harmothoe spinosa. a) extremo anterior en vista dorsal, b) parápodo medio, c) notoseta, d) neuroseta pectinada con el extremo distal bífido, d') detalle del extremo distal de la neuroseta, e) neuroseta con el extremo distal entero, f) élitro, g) papilas cónicas, h) papila filiforme, i) papila filiforme con el extremo distal globular liso, j) papila filiforme con el extremo distal globular espinoso. Harmothoe cf. exanthema: $\mathrm{k}$ ) extremo anterior en vista dorsal, l) parápodo medio, m) notoseta pectinada, n) neuroseta pectinada, o) élitro, p) papila cónica, q) papila digitiforme, r) macropapila globular. Halosydna patagonica: s) parápodo medio-posterior, t) notoseta distalmente aguzada con margen aserrado, u) notoseta distalmente redondeada con margen aserrado, v) neuroseta distalmente bidentada, w) papilas cónicas de los élitros.

Figure 4. Harmothoe spinosa. a) anterior end in dorsal view, b) middle parapodium, c) notoseta, d) pectinate neuroseta with bifurcate distal end, d') detail of distal end of a neuroseta, e) pectinate neuroseta with entire distal end, f) elytron, $g$ ) conical papillae, $\mathbf{h}$ ) filiform papilla, i) filiform papilla with smooth globular distal end, $\mathbf{j}$ ) filiform papilla with spinulose globular distal end. Harmothoe ef. exanthema: $\mathrm{k}$ ) anterior end in dorsal view, l) middle parapodium, $m$ ) pectinate notoseta, n) pectinate neuroseta , o) elytron, p) conical papilla, q) digitiform papilla, r) globular macropapilla. Halosydna patagonica: s) middle-posterior parapodium, t) distally pointed notoseta with serrated edge, $u$ ) distally rounded notoseta with serrated edge, $v$ ) distally bidentate neuroseta, w) conical papillae of the elytra. 
Observaciones: los ejemplares examinados coinciden parcialmente con la descripción de $H$. exanthema (Grube, 1856); se diferencia de ella, principalmente, en la morfología de las papilas globulares. Hartman (1964) señala la presencia de un filamento terminal mientras que en los élitros de los ejemplares examinados por nosotros se observan 12 filamentos, además de las micropapilas filiformes sobre la superficie que no son señaladas por esta autora. Debido a ello los ejemplares se determinaron como $H$. cf. exanthema.

Distribución geográfica: para $H$. exanthema, islas Falkland (Monro, 1930, 1936). En Chile, frente a Valparaíso (Grube, 1856); Calbuco, golfo de Ancud (Wesenberg-Lund, 1962), Aysén (este estudio) y estrecho de Magallanes (Ehlers, 1897, 1901a, Wesenberg-Lund, 1962).

\section{Harmothoe spinosa Kinberg, 1855}

(Figs. 4a-4j)

Harmothoe spinosa Kinberg, 1855: 386; 1910: 2122, pl. 6, fig. 31; Monro, 1930: 55-57; Fauvel, 1936: 6-7; Wesenberg-Lund, 1962: 22; Gallardo, 1977: 6870, fig. 3a-h; Rozbaczylo, 1985: 31-32; HartmannSchröder, 1986: 74; Hartmann-Schröder \& Rosenfeldt, 1988: 30-32, pl. 1-3, figs. 1-5.

Hermadion molluscum: Ehlers, 1900: 209; 1901a: 256; $1901 b: 43$.

Material examinado: Cimar-7: E-4 (1 ejemplar, SSUC 7117), E-26 (3 ejemplares, SSUC 7118). Cimar-8: E-4 (5 ejemplares, MNHNCL-An 2021), E-6 (1 ejemplar, MNHNCL-An 2022), E-59 (8 ejemplares, SSUC 7116); E-61 (4 ejemplares, SSUC 7119), E-63 (1 ejemplar, MNHNCL-An 2019), E71 (2 ejemplares, MNHNCL-An 2020).

Caracterización: el ejemplar de mayor tamaño mide $59 \mathrm{~mm}$ de longitud y $9 \mathrm{~mm}$ de ancho, con 36 setígeros. Prostomio más ancho que largo, con dos pequeñas proyecciones o picos en el margen anterior (Fig. 4a); con dos pares de ojos en el margen lateral de la región media y posterior del prostomio. Antena media delgada y papilada, con un largo ceratóforo; antenas laterales cortas y papiladas. Parápodos birramosos (Fig. 4b), con cirro dorsal más largo que el lóbulo parapodial, el ventral corto. Notosetas pectinadas (Fig. 4c). Neurosetas pectinadas con el extremo distal bífido (Figs. 4d y $4 d^{\prime}$ ) o entero (Fig. 4e). Dorso cubierto por 15 pares de élitros con el margen fimbriado (Fig. 4f); con papilas cónicas grandes cerca del margen (Figs. 4g y 4h), filiformes cortas con el extremo distal globular liso (Fig. 4i) y globular espinoso (Fig. 4j).

Distribución geográfica: islas Falkland (Fauvel, 1916); Nueva Zelanda (Benham, 1927); Antártica (Fauvel, 1936); islas Georgias del Sur (Ehlers, 1897); Kerguelen (Fauvel, 1936); islas Shetland del Sur, frente a isla Decepción, bahía Chile, isla Greenwich (Gallardo, 1977); Calbuco (Ehlers, 1901a), Aysén (este estudio) y estrecho de Magallanes (Kinberg, 1855).

\section{Polyeunoa dubia Hartmann-Schröder, 1965} (Figs. 5a-5f)

Polyeunoa dubia Hartmann-Schröder, 1965: 69-72, figs. 13-17; Rozbaczylo, 1985: 37.

Material examinado: Cimar-7: E-4 (18 ejemplares, SSUC 7120).

Caracterización: el ejemplar de mayor tamaño mide $79 \mathrm{~mm}$ de longitud y $6 \mathrm{~mm}$ de ancho, con 95 setígeros. Prostomio ovalado con dos pares de ojos (Fig. 5a); antena media larga y delgada; las laterales insertas ventralmente. Faringe con 18 papilas distales triangulares. Parápodos birramosos (Fig. 5b); el cirro dorsal sobrepasa al lóbulo parapodial y el ventral es tan largo como el lóbulo. Notosetas aciculares delgadas y con el margen finamente aserrado (Fig. 5c). Neurosetas distalmente enteras con una ligera expansión subdistal y margen aserrado (Fig. 5d). Dorso cubierto por 30 pares de élitros transparentes, ligeramente pigmentados hacia el centro del mismo (Fig. 5e), con papilas globulares pedunculadas (Fig. 5f).

Observaciones: en general, las características de los ejemplares examinados coinciden con las señaladas por Hartmann-Schröder (1965), sin embargo, las papilas de los élitros se observaron más globulares que las descritas por esta autora.

Distribución geográfica: Chile, golfo Corcovado (Hartmann-Schröder, 1965) y Aysén (este estudio).

\section{SIGALIONIDAE Malmgren}

\section{Leanira quatrefagesi Kinberg, 1855} (Figs. 5g-5k)

Leanira quatrefagesi Kinberg, 1855: 388; Ehlers, 1901b: 59, pl. 5, fig. 8; Monro, 1924: 46-47; 1936: 

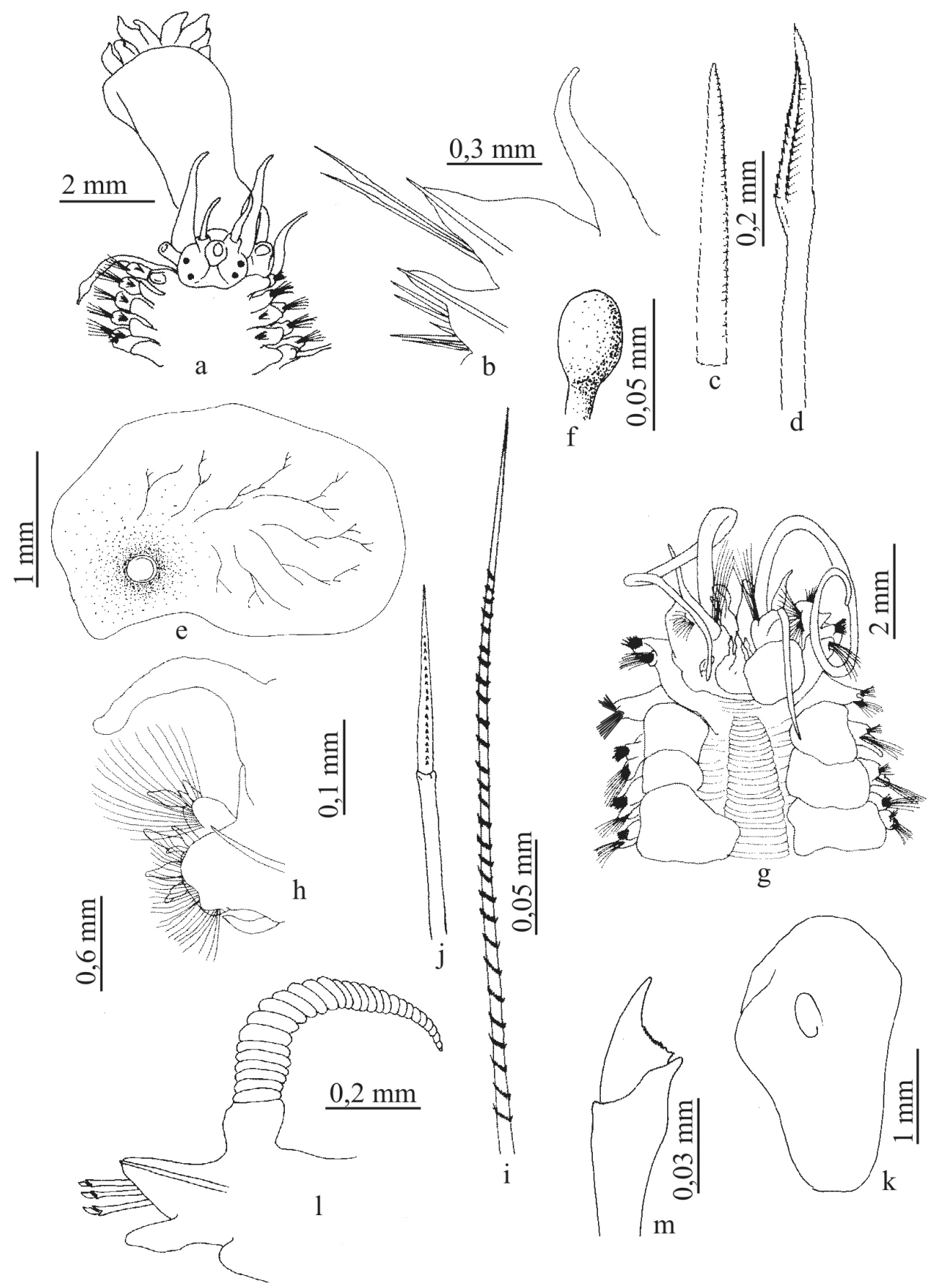

Figura 5. Polyeunoa dubia. a) extremo anterior en vista dorsal, b) parápodo medio, c) notoseta, d) neuroseta, e) élitro, f) papila del élitro. Leanira quatrefagesi: g) extremo anterior en vista dorsal, h) parápodo medio, i) notoseta, j) neuroseta, k) élitro; Syllidae indeterminado: l) parápodo, m) seta falciforme compuesta.

Figure 5. Polyeunoa dubia. a) anterior end in dorsal view, b) middle parapodium, c) notoseta, d) neuroseta, e) elytron, f) papilla of the elytron. Leanira quatrefagesi: g) anterior end in dorsal view, h) middle parapodium, i) notoseta, j) neuroseta, k) elytron; undetermined Syllidae: l) parapodium, m) compound falciger seta. 
105-106, fig. 13; Fauvel, 1941: 277-278; WesenbergLund, 1962: 27-28; Hartman, 1967: 40; Pettibone, 1970: 4-8, figs. 1-3; Rozbaczylo, 1985: 40.

Laenithalessa antennata Hartmann-Schröder, 1965 : 88-92, figs. 36-40.

Material examinado: Cimar-7: E-17a (7 ejemplares, SSUC 7121), E-21 (4 ejemplares, SSUC 7122), E-22 (2 ejemplares, SSUC 7123), E-24 (2 ejemplares, SSUC 7124), E-25 (1 ejemplar, SSUC 7125), E-32 (1 ejemplar, SSUC 7126), E-37 (3 ejemplares, SSUC 7127). Cimar-8: E-1 (1 ejemplar, MNHNCLAn 2027), E- 9 (10 ejemplares, MNHNCL-An 2025), E-51 (6 ejemplares, MNHNCL-An 2026), E53 (4 ejemplares, MNHNCL-An 2024), E-76 (1 ejemplar, MNHNCL-An 2023).

Caracterización: el ejemplar de mayor tamaño mide $52 \mathrm{~mm}$ de longitud y $8 \mathrm{~mm}$ de ancho. Prostomio ovalado; antena media más corta que el prostomio; las laterales insertas ventralmente, más cortas que la media (Fig. 5g). Los palpos son largos y extendidos hacia atrás alcanzan hasta el setígero siete. Sobre el ceratóforo dorsal se observan 1-2 estiloides muy cortos, ausentes en los ejemplares pequeños (10-28 mm). Primer setígero con setas capilares muy largas, dirigidas anteriormente. Parápodos birramosos con estiloides (Fig. 5h). Con branquias desde el setígero 23. Cirro parapodial ventral delgado y corto. Notosetas de dos tipos: capilares muy finas y capilares espinulosas (Fig. 5i). Neurosetas espiniformes compuestas (Fig. 5j). Los élitros (Fig. 5k), son ovalados, lisos y pequeños y no alcanzan a cubrir la superficie del animal.

Distribución geográfica: Atlántico sur frente al río de la Plata, Argentina e islas Falkland (Hartman, 1964); Sudáfrica; Pacífico sur frente a Chile, estrecho de Magallanes (Pettibone, 1970); Coquimbo; golfo de Ancud (Wesenberg-Lund, 1962); canal Messier, Aysén (McIntosh, 1885) e isla Hermite (Fauvel, 1941).

\section{SYLLIDAE Grube}

\section{Syllidae indeterminado \\ (Figs. 51-5m)}

Material examinado: Cimar-7: E-27 (1 ejemplar incompleto, SSUC 7128).

Caracterización: sólo se encontró un fragmento medio de un ejemplar. Cirro parapodial dorsal moniliforme (Fig. 51), con 18-28 artejos, de color violeta, alternándose uno largo y dos cortos. Setas falciformes compuestas (Fig. 5m).

Observaciones: el fragmento examinado no muestra suficientes caracteres taxonómicos que permitan asignarlo a alguna especie. Sin embargo, la forma aplanada o con forma de cinta de los segmentos parecen indicar que se trataría de una especie del género Trypanosyllis.

\section{NEREIDIDAE Johnston}

\section{Ceratocephale fauveli in Hartman, 1967 \\ (Figs. 6m-6p)}

Ceratocephale fauveli in Hartman, 1967: 62; Rozbaczylo, 1985: 83.

Material examinado: Cimar-7: E-41 (1 ejemplar, SSUC 7129).

Caracterización: ejemplar incompleto. Prostomio (Fig. 6m), con dos pares de ojos pequeños. Probóscide con tres papilas en hilera transversa sobre el área V y siete papilas largas en las áreas VII y VIII. Mandíbulas sin dientes. Cirro parapodial ventral simple en los dos primeros setígeros (Fig. 6n) y doble en los siguientes (Fig. 6o). Todas las notosetas y neurosetas son espiniformes homogonfas (Fig. 6p).

Distribución geográfica: Chile, frente a Valparaíso y Cabo de Hornos (Hartman, 1967); Aysén (este estudio).

\section{Nereis callaona (Grube, 1857)}

(Figs. 6a-6f)

Nereilepas callaona Grube, 1857: 165-166. Nereis callaona: Wesenberg-Lund, 1962: 75-76, fig. 28; Hartmann-Schröder, 1962a: 399-400; 1962b: 107; 1965: 297; Rozbaczylo \& Bolados, 1980: 209 212, figs. 2a-f; Rozbaczylo, 1985: 85.

Nereis robusta Kinberg, 1866a: 168-169; 1910: 50, pl. 20, fig. 1; Hartman, 1948: 64-65, pl. 10, figs. 1011; Wesenberg-Lund, 1962: 77.

Material examinado: Cimar-7: E-27 (3 ejemplares, SSUC 7130).

Caracterización: un ejemplar completo midió 32 $\mathrm{mm}$ de longitud y $3 \mathrm{~mm}$ de ancho. Prostomio (Fig. $6 a)$, con dos antenas y dos pares de ojos. Cuatro pares de cirros tentaculares, los dorsales más lar- 


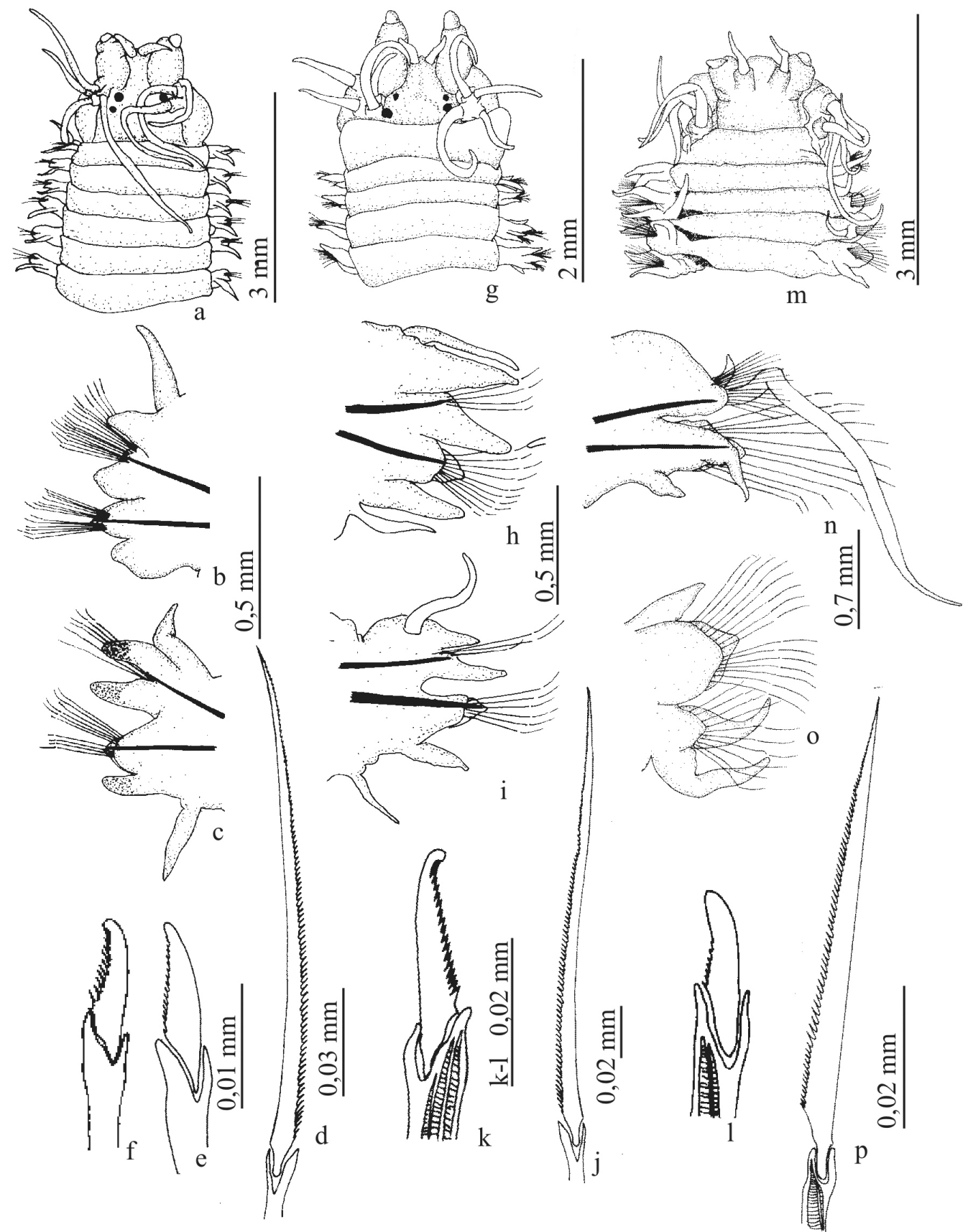

Figura 6. Nereis callaona. a) extremo anterior en vista dorsal, b) parápodo medio, c) parápodo posterior, d) notoseta espiniforme homogonfa, e) notoseta falciforme homogonfa, f) neuroseta falciforme heterogonfa. Nereis eugeniae: g) extremo anterior en vista dorsal, h) parápodo anterior, i) parápodo posterior, j) notoseta espiniforme homogonfa, k) neuroseta falciforme heterogonfa, l) notoseta falciforme homogonfa de los parápodos posteriores. Ceratocephale fauveli: $\mathbf{m}$ ) extremo anterior en vista dorsal, $\mathbf{n}$ ) parápodo anterior, o) parápodo posterior, $\mathbf{p}$ ) seta espiniforme homogonfa.

Figure 6. Nereis callaona. a) anterior end in dorsal view, b) middle parapodium, c) posterior parapodium, d) homogomph spiniger notoseta, e) homogomph falciger notoseta, f) heterogomph falciger neuroseta. Nereis eugeniae: g) anterior end in dorsal view, h) anterior parapodium, i) posterior parapodium, j) homogomph spiniger notoseta, k) homogomph falciger notoseta, l) homogomph falciger notoseta of posterior parapodia. Ceratocephale fauveli: $\mathbf{m}$ ) anterior end in dorsal view, $n$ ) anterior parapodium, o) posterior parapodium, p) homogomph spiniger. 
gos. Probóscide con paragnatos cónicos distribuidos de la siguiente manera: área $\mathrm{I}=2$ ó 3 ; área $\mathrm{II}=$ 20 , dispuestos en tres filas formando una banda oblicua; área III= aproximadamente 46, dispuestos formando un óvalo; área IV=28-30, dispuestos en forma triangular; área $\mathrm{V}=0$; área $\mathrm{VI}=4$, dispuestos en forma de cruz; áreas VII-VIII= con aproximadamente 60 , dispuestos en filas irregulares, alternándose paragnatos pequeños y grandes. Primer y segundo par de parápodos unirramosos, los restantes birramosos (Fig. 6b); en los posteriores los lóbulos están alargados y bien separados entre sí y los extremos distales están pigmentados (Fig. 6c). Notópodos anteriores con setas compuestas espiniformes homogonfas (Fig. 6d); los medios y posteriores, además de las espiniformes homogonfas llevan falciformes homogonfas (Fig. 6e). Neurópodos con falciformes heterogonfas (Fig. 6f) y espiniformes homogonfas.

Observaciones: esta especie ha sido registrada asociada a moluscos (fisurélidos), crustáceos (cirripedios) y equinodermos (Rozbaczylo \& Bolados, 1980).

Distribución geográfica: Panamá, costa del Atlántico (Fauchald, 1977b); islas Galápagos (Hartman, 1940); Perú, isla Chincha (Hartman, 1948); Chile, desde Iquique al estrecho de Magallanes (Wesenberg-Lund, 1962).

\section{Nereis eugeniae (Kinberg, 1866a)}

(Figs. 6g-61)

Nicon eugeniae Kinberg, 1866a: 178.

Nereis eugeniae: Ehlers, 1897: 67-70, pl. 4, figs. 94105; 1900: 214; 1901a: 259; 1901b: 105; WesenbergLund, 1962: 73-75, fig. 27; Hartmann-Schröder, 1962a: 400-404; 1965: 145; Rozbaczylo, 1985: 86.

Material examinado: Cimar-7: E-27 (2 ejemplares, SSUC 7131).

Caracterización: el ejemplar de mayor tamaño mide $30 \mathrm{~mm}$ de longitud y $3,5 \mathrm{~mm}$ de ancho (registros previos de Wesenberg-Lund (1962), señalan ejemplares de hasta $140 \mathrm{~mm}$ de longitud). Prostomio ancho, con dos pares de ojos (Fig. 6g). Cirros peristomiales cortos. Probóscide con paragnatos cónicos distribuidos de la siguiente manera: área $\mathrm{I}=$ 0; área II y IV= dos arcos irregulares; área $\mathrm{III}=3-5$, en fila transversal; área $\mathrm{V}=0$, área $\mathrm{VI}=$ pequeños paragnatos cónicos; áreas VII-VIII= paragnatos en filas transversales ampliamente separados.
Parápodos birramosos (Figs. 6h y 6i). Notópodos con lóbulos setígeros triangulares; neurópodos con un gran lóbulo redondeado. Parápodos anteriores con notosetas espiniformes homogonfas (Fig. 6j); las neurosetas superiores son espiniformes homogonfas y falciformes heterogonfas (Fig. 6k); las neurosetas inferiores son espiniformes y falciformes heterogonfas. Notópodos posteriores con 2 a 3 setas homogonfas falciformes con el artejo distal muy corto (Fig. 61).

Distribución geográfica: islas Falkland (Ramsay, 1914, Monro, 1930); Kerguelen (Monro, 1939); en Chile, seno de Reloncaví, isla Hoste (WesenbergLund, 1962) y estrecho de Magallanes (Kinberg, 1866a, Ehlers, 1897, 1901b).

\section{Platynereis australis (Schmarda, 1861)}

(Figs. 7a-7f)

Heteronereis australis Schmarda, 1861: 101-102, pl. 31, fig. 242.

Platynereis australis: Knox, 1951: 223-225, pl. 49, figs. 34-40; Hartmann-Schröder, 1962a: 427-432; 1962b: 108; 1965: 148; Day, 1967: 305, fig. 14.4.m; Imajima, 1972: 82-85, fig. 21a-o, fig. 22; Rozbaczylo \& Bolados, 1980: 216-218, fig. 4a-e; Rozbaczylo, 1985: 91-92; Knox \& Cameron, 1998: 57-58.

Platynereis magalhaensis Kinberg, 1866a: 177; 1910: 53, pl. 20, fig. 6; Monro, 1930: 106-107, fig. 37; Hartman, 1948: 60-61; Wesenberg-Lund, 1962: 85-88, figs. 33-34.

Material examinado: Cimar-7: E-27 (6 ejemplares, SSUC 7132). Cimar-8: E-67 (3 ejemplares, MNHNCL-An 2018).

Caracterización: el ejemplar de mayor tamaño mide $39 \mathrm{~mm}$ de longitud y $6 \mathrm{~mm}$ de ancho. El prostomio es más largo que ancho; con dos pares de ojos, el par anterior ligeramente más grande que el posterior (Fig. 7a). Un par de antenas frontales tan largas como los palpos, éstos últimos con palpostilos globulares. Cuatro pares de cirros tentaculares; los más largos extendidos hacia atrás alcanzan hasta el setígero 8-9. Probóscide con paragnatos pectinados distribuidos de la siguiente manera: áreas I, II y V= 0; área $\mathrm{III}=4-5$ hileras interrumpidas; área $\mathrm{IV}=8$ hileras dispuestas en forma de media luna; área VI= 2 hileras cortas, transversales; áreas VII-VIII $=5$ grupos transversales dispuestos en doble hilera. Los dos primeros pares de parápodos unirramosos, los siguientes birramosos (Fig. 7b). Notosetas 


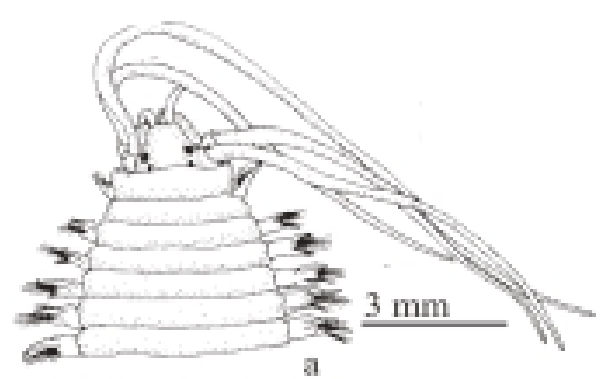

a
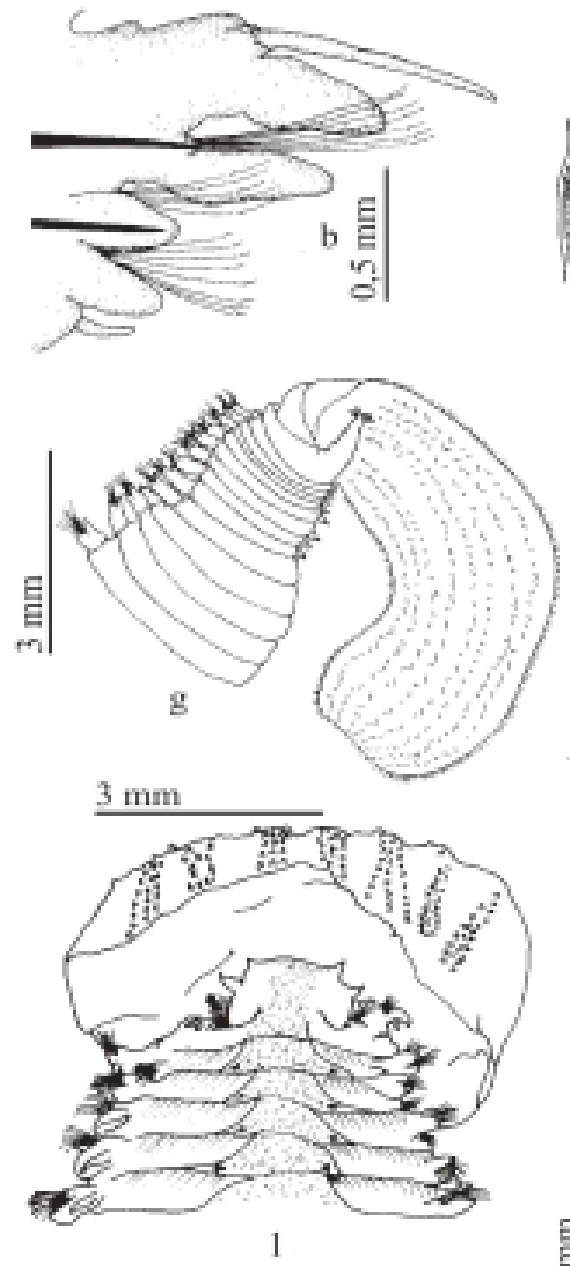

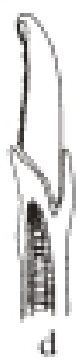

d

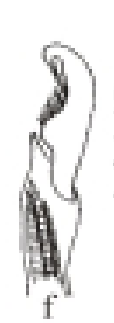

है
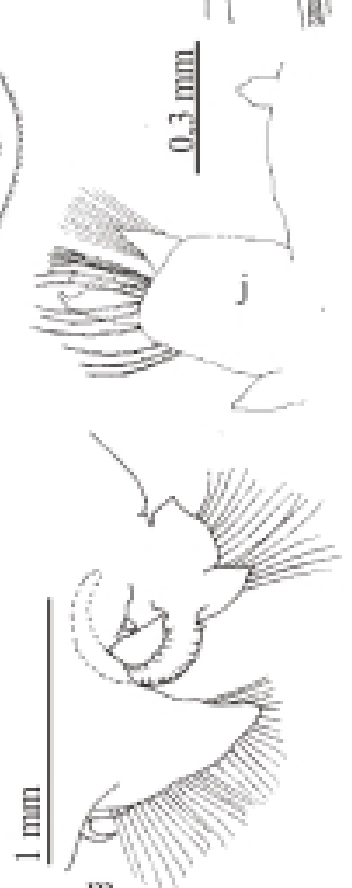
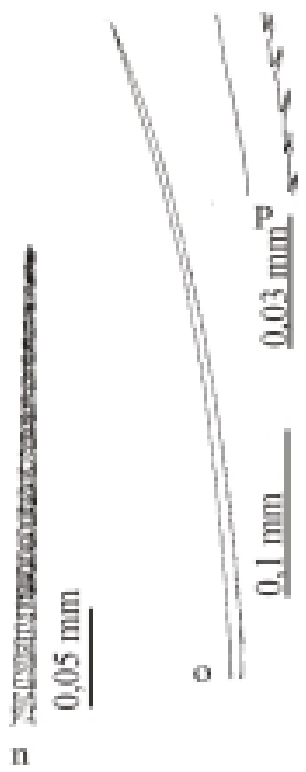

Figura 7. Platynereis australis. a) extremo anterior en vista dorsal, b) parápodo medio, c) notoseta espiniforme homogonfa, d) notoseta falciforme homogonfa, e) neuroseta espiniforme, f) seta falciforme heterogonfa. Glycera papillosa: g) extremo anterior en vista dorsal, h) papila proboscídea lisa alargada, i) papilas proboscídeas globulares, j) parápodo, k) neuroseta. Aglaophamus peruana: l) extremo anterior en vista dorsal, m) parápodo, n) seta barrada, o) seta capilar espinosa, p) detalle del margen de la seta capilar.

Figure 7. Platynereis australis. a) anterior end in dorsal view, b) middle parapodium, c) homogomph spiniger notoseta, d) homogomph falciger notoseta, e) spiniger neuroseta, f) homogomph falciger; Glycera papillosa: g) anterior end in dorsal view, h) elongated smooth proboscidial papilla i) globular proboscidial papillae, $\mathbf{j}$ ) parapodium, k) neuroseta. Aglaophamus peruana: l) anterior end in dorsal view, m) parapodium, n) barred seta, $o$ ) capillary seta, $p$ ) detail of the edge of a capillary seta. 
espiniformes homogonfas en los parápodos anteriores (Fig. 7c); en los posteriores, además de las espiniformes hay falciformes homogonfas (Fig. 7d). Neurosetas espiniformes homogonfas y falciformes heterogonfas (Fig. 7f) en el fascículo superior y espiniformes (Fig. 7e) y falciformes heterogonfas en el inferior.

Observaciones: Imajima (1972) señala que, en ejemplares adultos, las setas falciformes homogonfas pueden estar ausentes. La especie ha sido señalada principalmente para la región subantártica a excepción del registro de Imajima (1972) para Japón. Una de las características principales de esta especie es la presencia de largos cirros tentaculares así como la distribución de los paragnatos pectinados.

Distribución geográfica: Japón (Imajima, 1972); Georgia del Sur; islas Falkland (Monro, 1930); Kerguelen (McIntosh, 1885, Fauvel, 1950); mar de Ross (Benham, 1927); en Chile, desde Iquique hasta estrecho de Magallanes (Ehlers, 1900, 1901a, Wesenberg-Lund, 1962).

\section{GLYCERIDAE Grube}

\section{Glycera papillosa Grube, 1857} (Figs. 7g-7k)

Glycera papillosa Grube, 1857: 176-177; Ehlers, 1901b: 154; Kinberg, 1866b: 245; 1910: 58, pl. 21, fig. 3; Augener, 1922: 203-206, fig. 9a-c; Wesenberg-Lund, 1962: 103; Rozbaczylo, 1985: 103-104.

Material examinado: Cimar-7: E-27 (1 ejemplar, SSUC 7133).

Caracterización: el ejemplar mide $32 \mathrm{~mm}$ de longitud y $3 \mathrm{~mm}$ de ancho. Prostomio cónico con 8 anillos y cuatro pequeñas antenas en el extremo anterior (Fig. 7g). Probóscide con hileras de papilas lisas alargadas (Fig. 7h) y globulares (Fig. 7i). Parápodos (Fig. 7j), con dos lóbulos presetales: el superior pequeño y el inferior con extremo redondeado. Sin branquias. Notosetas simples capilares. Neurosetas espiniformes compuestas (Fig. 7k).

Observaciones: la especie es parecida a $G$. capitata Oersted, de la cual se diferencia por la forma del soporte mandibular siendo muy ahorquillado en $G$. papillosa.
Distribución geográfica: Carolina del Norte (Day, 1973); Sudáfrica (Day, 1967); Perú (Hartman, 1940); Chile, desde Arica hasta estrecho de Magallanes (Wesenberg-Lund, 1962) y archipiélago de Juan Fernández (Augener, 1922).

\section{NEPHTYIDAE Grube}

\section{Aglaophamus peruana (Hartman, 1940)}

(Figs. 71-7p)

Nephthys macroura peruana Hartman, 1940: 236, pl. 39, figs. 89-90, pl. 40, figs. 96-97.

Aglaophamus macroura: Hartmann-Schröder, 1965: 134-135, figs. 102-103.

Aglaophamus peruana: Hartman, 1967: 78; Rozbaczylo \& Castilla, 1974: 189; Rozbaczylo, 1985: 95.

Material examinado: Cimar-7: E-14 (1 ejemplar, SSUC 7134). Cimar-8: E-6 (1 ejemplar, MNHNCLAn 2015), E-54 (1 ejemplar, MNHNCL-An 2016), E-71 (1 ejemplar, SSUC 7135), E-78 (1 ejemplar, MNHNCL-An 2017).

Caracterización: el ejemplar de mayor tamaño mide $52 \mathrm{~mm}$ de longitud y $7 \mathrm{~mm}$ de ancho, con 93 setígeros. Prostomio con forma trapezoidal (Fig. 71); ojos ausentes; con un par de antenas anterolaterales y un par ventrolaterales. Sobre la superficie corporal dorsal se extiende una banda longitudinal de color ocre desde el extremo anterior del prostomio hasta los segmentos posteriores. Faringe globular con 22 papilas bífidas en el extremo distal, las medio dorsales y medio ventrales más pequeñas. Sobre la superficie de la probóscide se disponen 22 hileras longitudinales de papilas cónicas, cada una de ellas, a su vez, con 2 ó 3 hileras irregulares de papilas. La superficie proximal de la probóscide es lisa. Parápodos birramosos (Fig. 7m); setas barradas (Fig. 7n), y capilares espinosas (Figs. 7o-7p). No presenta setas liradas.

Distribución geográfica: Perú (Hartman, 1940); en Chile, Valparaíso a Metalqui, Chiloé (HartmannSchröder, 1965); Aysén (este estudio).

\section{DISCUSIÓN}

El conocimiento general sobre la riqueza específica de poliquetos bentónicos de la región de Aysén, proviene principalmente de las publicaciones de Wesenberg-Lund (1962), Hartmann-Schröder 
(1962b, 1965) y Cañete et al. (1999), con un total de 57 especies registradas en profundidades menores a $30 \mathrm{~m}$, de las cuales 28 especies $(49,1 \%)$, pertenecen al clado Phyllodocida. La Lund University Chile Expedition 1948-1949, recorrió desde Boca del Guafo (433's' hasta el canal Errázuriz, Faro Islote Diego ( $45^{\circ} 39^{\prime}$ 'S) en profundidades de 0-25 m, recolectando un total de 15 especies (WesenbergLund, 1962). Hartmann-Schröder (1962b) describió cuatro especies nuevas de un total de siete especies de poliquetos asociados a Macrocystis pyrifera

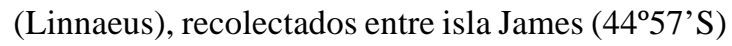
e isla Melchor (45 $10^{\circ}$ 'S). Posteriormente, HartmannSchröder (1965) da a conocer 20 especies de poliquetos, de las cuales siete y una subespecie son nuevas para la ciencia, provenientes del archipiélago de los Chonos (isla James, Puerto Aguirre, isla Melchor), asociadas principalmente a bancos de mitílidos. Cañete et al. (1999) estudiaron los poliquetos bentónicos de tres localidades submareales someras del fiordo Aysén, entre bahía Acantilada, desembocadura del río Cóndor y desembocadura del río Cuervo ( $42^{\circ} 22^{\prime}$ a $\left.45^{\circ} 20^{\prime} \mathrm{S}\right)$, registrando un total de 38 especies.

La información disponible permite aumentar la representación de poliquetos bentónicos submareales a un total de 66 especies en la región de Aysén, producto de la incorporación de nueve nuevos registros de especies del clado Phyllodocida obtenidos en este estudio, de las cuales cinco pertenecen a la familia Polynoidae (A. antarctica, E. lobulata, E. patagonica, H. spinosa y $P$. dubia), dos a Nereididae (C. fauveli y $N$. callaona), y uno a Aphroditidae (A. bisetosa) y Sigalionidae ( $L$. quatrefagesi), respectivamente, permitiendo aumentar a 37 especies $(56,1 \%)$ la representación del clado en la fauna de poliquetos bentónicos de la región de Aysén.

Los cruceros Cimar-7 y Cimar-8 Fiordos mediante la realización de estudios bentónicos a profundidades mayores a $30 \mathrm{~m}$ en el área, han permitido ampliar los rangos de distribución batimétrica de $H$. patagonica, P. australis, G. papillosa y A. peruana, que habitualmente habían sido registradas en las zonas intermareal y submareal somera (ver WesenbergLund, 1962; Hartmann-Schröder, 1965; Cañete et al. 1999), a profundidades entre 110 y $246 \mathrm{~m}$.

Los patrones de distribución geográfica de los taxa del clado Phyllodocida permiten definir que las especies A. antarctica, E. lobulata, E. patagonica, H. spinosa, P. dubia y N. eugeniae están restringi- das a aguas templadas frías dentro la Provincia Magallánica, alcanzando en general como límite más septentrional de distribución la zona de Chiloé ( $c a$. $41^{\circ} \mathrm{S}$ ). Por otro lado, A. bisetosa, $H$. patagonica, $H$. cf. exanthema, L. quatrefagesi, C. fauveli, N. callaona, P. australis, G. papillosa y A. peruana presentan una amplia distribución a lo largo de la costa de Chile, abarcando principalmente desde aguas templadas cálidas ( $\left.c a .18^{\circ} 20^{\prime} \mathrm{S}\right)$ de la Provincia Peruana a templadas frías $\left(c a .53^{\circ}-55^{\circ} \mathrm{S}\right)$ de la Provincia Magallánica.

Con la realización de los cruceros Cimar-7 y Cimar-8 Fiordos, aumentan a seis las expediciones con información sobre poliquetos realizadas hasta el momento en esta región del Pacífico suroriental, permitiendo configurar una visión general del número total de clados de poliquetos bentónicos presentes en la región de Aysén, estableciéndose hasta ahora la presencia de seis clados, Phyllodocida, Terebellida, Eunicida, Scolecida, Sabellida y Spionida en el mar interno de la región.

Finalmente, los cruceros Cimar-7 y Cimar-8 Fiordos se han constituido en pioneros en realizar estudios bentónicos a profundidades mayores a 30 $\mathrm{m}$ en el área, permitiendo aumentar el conocimiento de la biodiversidad marina, en términos de ampliación de rangos geográficos y batimétricos $(\sim 450$ m) de taxa de poliquetos bentónicos submareales.

\section{AGRADECIMIENTOS}

Los autores agradecen al Comité Oceanográfico Nacional por el apoyo logístico prestado para la realización de los cruceros Cimar 7 y Cimar 8-Fiordos. También agradecemos a Patricio Zavala por la colaboración técnica en la catalogación y posterior almacenamiento de los taxa de poliquetos bentónicos en la sala "Colección de Flora y Fauna Profesor Patricio Sánchez Reyes", Pontificia Universidad Católica de Chile y en el Museo Nacional de Historia Natural. Hacemos extensivos nuestros agradecimientos a tres revisores anónimos por sus valiosas sugerencias y comentarios críticos.

\section{REFERENCIAS}

Augener, H. 1922. Litorale Polychaeten von Juan Fernández. En: C. Skottsberg (ed.). The Natural History of Juan Fernández and Easter Island, 3: 161-218. 
Benham, W.B. 1927. Polychaeta. British Antarctic Terra Nova Expedition, 1910. Natural History Reports, Zoology, 7(2): 47-182.

Bergström, E. 1916. Die Polynoiden der schwedischen Südpolar-Expedition 1901-1903. Zool. Bidr. Uppsala, 4: 249-304.

Cañete, J.I., G.L. Leighton \& F.F. Aguilera 1999. Polychaetes from Aysén Fjord, Chile: distribution, abundance and biogeographical comparison with the shallow soft-bottom polychaete fauna from Antarctic and the Magellan Province. Sci. Mar., 63(1): 243-252.

Day, J.H. 1967. A monograph on the Polychaeta of Southern Africa. British Museum Nat. Hist. Publ., 656: $878 \mathrm{pp}$.

Day, J.H. 1973. New Polychaeta from Beaufort, with a key to all species recorded from North Carolina. NOAA Technical Rep. NMFS Circ-375: 140 pp.

Ehlers, E. 1897. Polychaeten. Hamburger Magalhaenischen Sammelreise. Hamburg, 148 pp.

Ehlers, E. 1900. Magellanische Anneliden gesammelt während der schwedischen Expedition nach den Magellansländern. Nachr. K. wiss. Göttingen, 1900: 206-223.

Ehlers, E. 1901a. Die Anneliden der Sammlung Plate. Fauna Chilensis Zool. Jahrb., Suppl., 5: 251-272.

Ehlers, E. 1901b. Die Polychaeten des magellanischen und chilenischen Strandes. Ein faunistischer Versuch. Festschrift zur Feier des Hundertfünfzigjährigen Bestehens der Königlichen Gesellschaft der Wissenschaften zu Göttingen. (Abh. Math.-Phys. K.). Berlin, 232 pp.

Fauvel, P. 1916. Annélides polychètes des Iles Falkland recueillies par M. Rupert Vallentin Esqre (1902-1910). Arch. Zool. Exp. Gen. Paris, 55: 417 482.

Fauvel, P. 1936. Polychètes Expédition antarctique Belgica. Résultats du voyage de la Belgica en 18971899, sous le commandement de A. de Gerlache de Gomery. 46 pp.

Fauvel, P. 1941. Annélides polychètes de la Mission du Cap Horn (1882-1883). Bull. Mus. Hist. Nat. Paris, sér. 2, 13(4): 272-298.

Fauvel, P. 1950. Missions du Bariment Polaire «Commandant-Charcot». Récoltes faites en Terre
Adèlie (1950). Bull. Mus. Hist. Nat. Paris, sér. 2, 22: 753-773.

Fauchald, K. 1977a. The polychaete worms: definitions and keys to the orders, families and genera. Nat. Hist. Mus. Los Angeles County. Sci. Ser., 28: 1-190.

Fauchald, K. 1977b. Polychaetes from intertidal areas in Panama, with a review of previous shallow-water records. Smithsonian Contr. Zool., 221: 1-81.

Fauchald, K. \& G.W. Rouse. 1997. Polychaete systematics: past and present. Zool. Scr., 26: 71138.

Gallardo, V.A. 1977. Polynoidae collected during the XXII Chilean Antarctic Expedition, 1967-1968. En: D.J. Reish \& K. Fauchald (eds.). Essays on polychaetous annelids in Memory of Dr. Olga Hartman. Allan Hancock Foundation, University of Southern California, Los Angeles, pp. 63-83.

Glasby, K., P. Hutchings, K. Fauchald, H. Paxton, G. Rouse, C. Watson-Russel \& R. Wilson. 2000. Class Polychaeta. En: P.L. Beesley, G.J.B. Ross \& C.J. Glasby (eds.). Polychaetes \& allies: the Southern synthesis. Fauna of Australia. Vol. 4A. Polychaeta, Myzostomida, Pogonophora, Echiura, Sipuncula. CSIRO Publishing, Melbourne, pp. 1296.

Grube, A.E. 1856. Annulata Oerstediana. Enumeratio Annulatorum, quae in itinere per Indiam Occidentalem et American Centralem annis 18451848 suscepto legit. cl. A. S. Oersted, adjectis speciebus nonnullis a cl. H. Kröyer in itinere ad American meridionalem collectis. Pt.1. Vidensk. Medd. naturh. Foren Kjöbenhavn, 1856: 44-62.

Grube, A.E. 1857. Annulata Oerstediana, etc. Pt. 2. Vidensk. Medd. Naturh. Foren. Kjöbenhavn, 1857: 158-186.

Hartman, O. 1940. Polychaetous annelids. II. Chrysopetalidae to Goniadidae. Allan Hancock Pacific Exped., 7(3): 173-287.

Hartman, O. 1948. The marine annelids erected by Kinberg with notes on some other types in the Swedish State Museum. Ark. Zool., 42A(1): 1-137.

Hartman, O. 1953. Non-pelagic polychaeta of the Swedish Antarctic Expedition 1901-1903. Further zoological results of the Swedish Antarctic Expedition 1901-1903, 4(2): 1-83. 
Hartman, O. 1964. Polychaeta Errantia of Antarctica. Antarctic Res. Ser., 3: 1-131.

Hartman, O. 1967. Polychaeta annelids collected by the USNS Eltanin and Staten Island Cruises, chiefly from Antarctic Seas. Allan Hancock Monogr. Mar. Biol., 2: 1-387.

Hartmann-Schröder, G. 1962a. Zur Kenntnis der Nereiden Chiles (Polychaeta errantia), mit Beschreibung epitoker Stadien einiger Arten und der Jugendentwicklung von Perinereis vallata (Grube). Zool. Anz., 168(11-12): 389-441.

Hartmann-Schröder, G. 1962b. Zur Kenntnis des Eulitorals der chilenischen Pazifikküste und der argentinischen Küste Südpatagoniens unter besonderer Berücksichtigung der Polychaeten und Ostracoden. T1.II. Die Polychaeten des Eulitorals. Mitt. Hamburg. Zool. Mus. Inst., 60(Suppl.): 57167.

Hartmann-Schröder, G. 1965. Zur Kenntnis des Sublitorals der chilenischen Küste unter besonderer Berücksichtigung der Polychaeten und Ostracoden. T1. II. Die Polychaeten des Sublitorals. Mitt. Hamburg. Zool. Mus. Inst., 62(Suppl.): 59-305.

Hartmann-Schröder, G. 1986. Die Polychaeten der 56. Reise der "Meteor" zu den South ShetlandInseln (Antarktis). Mitt. Hamb. Zool. Mus. Inst., 83: 71-100.

Hartmann-Schröder, G. \& P. Rosenfeldt. 1988. Die Polychaeten der "Polarstern"-Reise ANT III/2 in die Antarktis 1984. Teil 1. Euphrosinidae bis Chaetopteridae. Mitt. Hamb. Zool. Mus. Inst., 85: 25-72.

Imajima, M. 1972. Review of the annelid worms of the family Nereidae of Japan, with descriptions of five new species or subspecies. Bull. Natn. Sci. Mus. Tokyo, 15(1): 37-153.

Kinberg, J.G.H. 1855. Nya slägten och arter af Annelider. Öfv. Svenska Vetensk. Akad. Förh., 12: 381-388.

Kinberg, J.G.H. 1858-1910. Annulater. En: Kongliga Svenska Fregatten Eugenies Resa omkring jorden under befäl af C. A. Virgin aren 1851-1853. Vetenskapliga lakttagelser pa Konung Oscar den Förstes befallning utgifna af K. Svenska Vetenskapsakademien. Almquist \& Wiksells, Stockholm, Zoologi, 3: 1-78.
Kinberg, J.G.H. 1866a. Annulata nova. Öfv. Svenska Vetensk. Akad. Förh., 22: 167-179.

Kinberg, J.G.H. 1866b. Annulata nova. Öfv. Svenska Vetensk. Akad. Förh., 22: 239-258.

Knox, G.A. 1951. The polychaetous annelids of Banks Peninsula. Rec. Cant. Mus., 5 (5): 213-229.

Knox, G.A. 1960. Biological results of the Chatham Islands 1954 Expedition. Pt. 3. Polychaeta Errantia. Mem. New Zealand Ocean. Inst., 6: 77-143.

Knox, G.A. \& D.B. Cameron. 1998. The marine fauna of the Ross Sea: Polychaeta. Wellington: NIWA Biodiversity Memoir, 108: 1-125.

McIntosh, W.C. 1885. Report on the Annelida Polychaeta collected by H.M.S. Challenger during the years 1873-1876. Challenger Rep., 12: 1-554.

Monro, C.C.A. 1924. On the polychaeta collected by H.M.S. "Alert” 1881-1882. Families Polynoidae, Sigalionidae, Eunicidae. J. Linn. Soc., London, 36: 37-77.

Monro, C.C.A. 1930. Polychaete worms. Discovery Rep., 2: 1-222.

Monro, C.C.A. 1936. Polychaete worms II. Discovery Rep., 12: 59-198.

Monro, C.C.A. 1939. Polychaeta. Antarctic research expedition 1929-1931 BANZARE Rep., Ser. B (Zoology and Botany), 4(4): 87-156.

Pettibone, M.H. 1970. Revision of some species referred to Leanira Kinberg (Polychaeta: Sigalionidae). Smithsonian Contr. Zool., 53: 1-25.

Ramsay, L.N.G. 1914. Polychaeta of the family Nereidae, collected by the Scottish National Antarctic Expedition (1902-1904). Trans. R. Soc. Edinb., 50(3): 41-48.

Rouse, G.W. \& K. Fauchald. 1997. Cladistics and polychaetes. Zool. Scr., 26: 139-204.

Rouse, G.W. 2000. Phyllodocida. En: P.L. Beesley, G.J.B. Ross \& C.J. Glasby (eds.). Polychaetes and Allies: the Southern synthesis. Fauna of Australia. Vol. 4A. Polychaeta, Myzostomida, Pogonophora, Echiura, Sipuncula. CSIRO Publishing, Melbourne, $112 \mathrm{pp}$.

Rouse, G.W. \& F. Pleijel. 2001. Polychaetes. Oxford University Press, Oxford, 1-354. 
Rozbaczylo, N. 1980. Clave para el reconocimiento de familias de Anélidos Poliquetos del mar chileno. Stud. Neotrop. Fauna Environ., 15(3-4): 167196.

Rozbaczylo, N. 1985. Los anélidos poliquetos de Chile. Indice sinonímico y distribución geográfica de especies. Ediciones Pontificia Universidad Católica de Chile. Monogr. Biol., 3: 1-284.

Rozbaczylo, N. \& E. Canahuire. 2000. Aphrodita bisetosa (Polychaeta: Aphroditidae), a new species of sea mouse from the southeastern Pacific Ocean off central Chile. Proc. Biol. Soc. Wash., 113(2): 493-499.

Rozbaczylo, N. \& J. Bolados. 1980. Nereidos de Iquique, Chile. Bol. Mus. Nac. Hist. Nat. Chile, 37: 205-224.

Recibido: 29 noviembre 2004; Aceptado: 14 abril 2005
Rozbaczylo, N. \& J.C. Castilla. 1974. La familia Nephtyidae en Chile (Annelida, Polychaeta). Stud. Neotrop. Fauna, 9(2): 179-206.

Schmarda, L.K. 1861. Neue wirbellose thiere beobachtet und gesammelt auf einer Reise um die Erde 1853 bis 1857. 1: Turbellarien, Rotatorien und Anneliden, 2: 1-164.

Seidler, H.J. 1922. Ueber Branchialfortsätze bei Polynoiden, nebst Beschreibung einer neuen Art (Physalidonotus lobulatus). Sitzber. Ges. naturf. Freunde Berlin, 1921: 86-91.

Seidler, H.J. 1924. Beiträge zur Kenntnis der Polynoiden I. Arch. Natugesch., 80A(11): 1-217.

Wesenberg-Lund, E. 1962. Polychaeta Errantia. Rep. Lund. Univ. Chile Exped. (43). Acta Univ. Lund, 57(12): 1-139. 